\title{
Intracellular speciation of gold nanorods alters the conformational dynamics of genomic DNA
}

\author{
Diwei Ho ${ }^{1,12}$, Jessica A. Kretzmann 1,12, Marck Norret ${ }^{1}$, Priyanka Toshniwal', Jean-Pierre Veder ${ }^{2}$, \\ Haibo Jiang1,3, Paul Guagliardo3, Alaa M. Munshi'111, Reena Chawla ${ }^{4,5,6}$, Cameron W. Evans', \\ Tristan D. Clemons ', Michelle Nguyen ${ }^{1}$, Amy L. Kretzmann', Amanda J. Blythe', Martin Saunders ${ }^{1,3}$, \\ Michael Archer7, Melinda Fitzgerald ${ }^{7,8,9}$, Jeffrey A. Keelan ${ }^{10}{ }^{10}$, Charles S. Bond (1) ${ }^{1}$, Matt R. Kilburn $\mathbb{1 0}^{3}$, \\ Laurence H. Hurley ${ }^{4,5,6}$, Nicole M.Smith ${ }^{1 \star}$ and K. Swaminathan Iyer ${ }^{1 \star}$
}

\begin{abstract}
Gold nanorods are one of the most widely explored inorganic materials in nanomedicine for diagnostics, therapeutics and sensing ${ }^{1}$. It has been shown that gold nanorods are not cytotoxic and localize within cytoplasmic vesicles following endocytosis, with no nuclear localization ${ }^{2,3}$, but other studies have reported alterations in gene expression profiles in cells following exposure to gold nanorods, via unknown mechanisms ${ }^{4}$. In this work we describe a pathway that can contribute to this phenomenon. By mapping the intracellular chemical speciation process of gold nanorods, we show that the commonly used Au-thiol conjugation, which is important for maintaining the noble (inert) properties of gold nanostructures, is altered following endocytosis, resulting in the formation of Au( $(1)$-thiolates that localize in the nucleus ${ }^{5}$. Furthermore, we show that nuclear localization of the gold species perturbs the dynamic microenvironment within the nucleus and triggers alteration of gene expression in human cells. We demonstrate this using quantitative visualization of ubiquitous DNA G-quadruplex structures, which are sensitive to ionic imbalances, as an indicator of the formation of structural alterations in genomic DNA.
\end{abstract}

Traditionally, gold nanorods (GNRs) are synthesized with a non-covalently bound bilayer of cetyltrimethylammonium bromide (CTAB) that dissociates from the GNR surface under physiological conditions, resulting in significant cytotoxicity. This can be overcome by exchanging CTAB with a thiolated analogue-(16-mercaptohexadecyl)trimethylammonium bromide (MTAB) - to enable covalent interaction between the gold surface and pendant thiol groups resulting in highly efficient non-toxic cellular internalization ${ }^{6}$. In this study, we synthesized two distinct MTAB-modified GNRs of different lengths $(54.1 \pm 5.2 \mathrm{~nm}$ and $91.8 \pm 11.3 \mathrm{~nm})$ and identical diameters $(19.0 \pm 2.0 \mathrm{~nm}$ and $18.9 \pm 2.4 \mathrm{~nm})$ with corresponding aspect ratios (ARs) of 2.8 and 4.9, respectively (Fig. 1b-d). We also synthesized MTAB-coated gold nanoparticles (GNPs, Fig. 1a) of similar diameter to the GNRs as a control to evaluate shapedependent contributions. The complete exchange of CTAB for its thiolated analogue MTAB was validated by the detection of sulfur using electron energy loss spectra (EELS) (Supplementary Fig. 1). The zeta potentials of the GNPs/GNRs were $32.8 \pm 0.6$ (GNP), $36.8 \pm 0.6$ (AR 2.8) and 25.8 \pm 1.2 (AR 4.9) (Supplementary Fig. 2). The UV-vis absorbance of the GNRs demonstrated a characteristic redshift in the longitudinal surface plasmon resonance (LSPR) from 679 to $953 \mathrm{~nm}$ for the short (NRS) and long (NRL) nanorods, respectively (Fig. 1e). The presence of the sharp LSPR for the GNRs (full-width at half-maximum $(\mathrm{FWHM})=106.9 \mathrm{~nm}(\mathrm{AR} 2.8)$ and $195.0 \mathrm{~nm}$ (AR 4.9)) is indicative of their narrow size distribution. Both GNR samples had negligible amounts of shape impurities (for example, spheres) (Fig. 1b,c).

We next evaluated the toxicity profile and cellular internalization of the GNRs in HEK-293T and MCF-7 cells. The toxicity profiles included MTAB-coated GNRs and GNPs with CTAB-coated GNRs and GNPs as controls to analyse surface contributions, CTAB and MTAB controls (assessing for half maximum inhibitory concentration $\left(\mathrm{IC}_{50}\right)$, Supplementary Fig. 3) to analyse surfactant contributions and $25 \mathrm{~nm}$ polystyrene nanoparticles (PS NPs) as a gold-free control. No changes in cell viability were observed on exposure to either MTAB-coated GNRs or GNPs at concentrations up to $100 \mu$ $\mathrm{g} \mathrm{ml}^{-1}$ (Fig. 1f,g). The only observable toxicity was associated with CTAB-coated GNRs and GNPs, in accordance with previous studies $^{7,8}$. We also observed significantly higher rate of internalization for the MTAB-GNRs compared to CTAB-GNRs, as previously reported (Fig. 1h) ${ }^{6}$. Finally, the cytoplasmic localization of MTABGNRs was confirmed by TEM analysis of both MCF-7 and HEK293T cells (Fig. 1i-1), with no observable alterations in GNR and GNP morphology, consistent with previous reports ${ }^{2,6}$.

Biophysical and computational analyses of cationic gold nanoparticles have shown them to induce DNA bending and strand separation in an extracellular setting 9 . However, alterations in gene expression profiles following internalization of GNRs has also been reported, despite the fact that GNRs are localized in the cytoplasm with no genomic DNA interaction. Gene expression profiles are highly dependent on complex interactions between DNA-binding

'School of Molecular Sciences, The University of Western Australia, Perth, Western Australia, Australia. ${ }^{2} J o h n$ de Laeter Centre, Curtin University, Perth, Western Australia, Australia. ${ }^{3}$ Centre for Microscopy, Characterisation and Analysis, The University of Western Australia, Perth, Western Australia, Australia. ${ }^{4}$ College of Pharmacy, University of Arizona, Tucson, AZ, USA. ${ }^{5} \mathrm{BIO} 5$ Institute, University of Arizona, Tucson, AZ, USA. ${ }^{6}$ Arizona Cancer Center, University of Arizona, Tucson, AZ, USA. ${ }^{7}$ School of Biological Sciences, The University of Western Australia, Perth, Western Australia, Australia. ${ }^{8}$ Curtin Health Innovation Research Institute, Curtin University, Perth, Western Australia, Australia. ${ }^{9}$ Perron Institute for Neurological and Translational Science, Sarich Neuroscience Research Institute, Perth, Western Australia, Australia. ${ }^{10}$ Schools of Obstetrics \& Gynaecology and Biomedical Sciences, The University of Western Australia, Perth, Western Australia, Australia. "Present address: Department of Chemistry, Faculty of Applied Science, Umm Al-Qura University, Makkah, Saudi Arabia. ${ }^{12}$ These authors contributed equally: Diwei Ho, Jessica A. Kretzmann. *e-mail: nicole.smith@uwa.edu.au; swaminatha.iyer@uwa.edu.au 

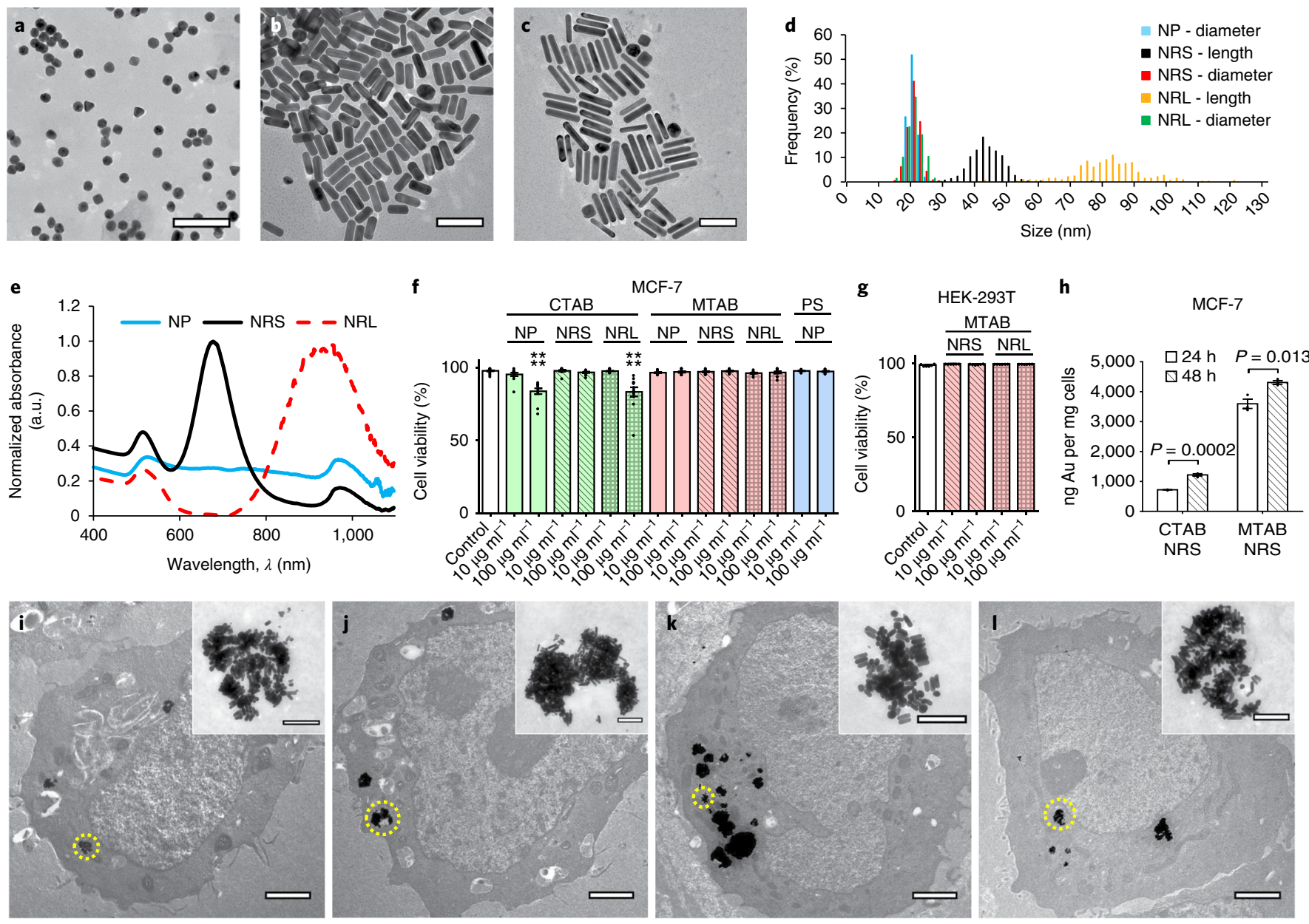

Fig. 1 | Thiol-functionalized gold nanorods are non-toxic and are localized in intracellular vesicles following endocytosis. Gold nanomaterial characterization and cytotoxicity studies. a-c, Transmission electron microscopy (TEM) images of gold nanomaterials at ARs of 1.0 (a), 2.8 (b) and 4.9 (c). d, Size distribution of AR 1.0 (NP), AR 2.8 (NRS) and AR 4.9 (NRL) gold nanomaterials as assessed by TEM. e, UV-vis spectroscopy of MTAB-NP/ NRS/NRL. $\mathbf{f}, \mathbf{g}$, Live/dead cell viability assays on MCF-7 (f, $n=9$ per condition) and HEK-293T ( $\mathbf{g}, n=6$ per condition) cells at 10 and $100 \mu g \mathrm{ml}^{-1}$ for CTABand MTAB-coated gold nanomaterials after $48 \mathrm{~h}$. One-way analysis of variance (ANOVA) was performed for $\mathbf{f}$ and $\mathbf{g}$ ( $\left.{ }^{\star \star \star \star} P<0.0001\right)$. $\mathbf{h}$, Concentration of NRS associated with cells after 24 and $48 \mathrm{~h}$, assessed by inductively coupled plasma-mass spectroscopy (ICP-MS) ( $n=3$ per condition per time point). Statistical analysis was performed with two-tailed unpaired $t$-test between 24 and $48 \mathrm{~h}$ for each NRS. $\mathbf{i}-\mathbf{I}$, TEM images of NRS (i,k) and NRL

(j, I) internalization in HEK-293T (i,j) and MCF-7 (k, I) cells at $100 \mu \mathrm{g} \mathrm{ml}^{-1}$. Insets, Regions of cytoplasmic GNRs at high magnification, indicated by yellow dashed circles. $\mathbf{i}-\mathbf{I}$ were repeated twice independently with similar results. All data in graphs are represented as mean \pm s.e.m. and individual data points are shown as dots. Scale bars, $100 \mathrm{~nm}$ (a-c); $2 \mu \mathrm{m}$ (i-I); $200 \mathrm{~nm}$ (i-I insets).

proteins, genomic DNA sequences and the signals transmitted between the cytoplasm to the nucleus, which are dynamic and reciprocal. Besides these sequence-specific interactions, it is now believed that sequence-dependent structural dynamics of DNA also play a significant role in transcription ${ }^{10,11}$. The stability of these sequence-dependent dynamic structures of DNA are highly dependent on the immediate ionic microenvironment ${ }^{12}$. For example, certain G-rich DNA sequences have been shown to fold into noncanonical four-stranded structures called G-quadruplexes (G4, which are stabilized by cations) in the regulatory regions of the human genome and are proven to regulate transcription, recombination and replication ${ }^{12-14}$. To determine whether exposure of cells to GNRs can result in structural alterations of genomic DNA, we evaluated alterations in G4 foci in cells exposed to GNRs and GNPs (CTAB- and MTAB-coated), compared to PS NPs, MTAB only, CTAB only, chloro(triphenylphosphine) gold(I) $\left(\mathrm{PPh}_{3} \cdot \mathrm{AuCl}\right)$ treated and untreated cells. Quantitative visualization of G4 foci was conducted via immunohistochemistry with a G4-structure-specific antibody (Fig. 2a-c,g-i) ${ }^{15}$. A statistically significant decrease in G4 foci was observed for AR 2.8 MTAB-GNRs at $100 \mu \mathrm{g} \mathrm{ml}^{-1}$ in both HEK-293T (-44.8\%) and MCF-7 (-38.3\%) cells; for AR 4.9 MTABGNRs and MTAB-GNPs, a significant decrease in G4 foci was only observed in MCF-7 cells (-48.0\%) when compared to controls (Fig. 2m,o; Supplementary Fig. 4). Importantly, $\mathrm{PPh}_{3}$. AuCl treatment in MCF-7 cells also demonstrated a statistically significant decrease in G4 foci $(-15.3 \%)$. Given that G4-DNA are dynamic structures sensitive to changes in the ionic microenvironment within the nucleus, we next tested if these observed cytoplasmic GNR-induced changes in G4 foci can be reversed. We thus incubated both HEK-293T and MCF-7 cells post-GNR internalization with a high-specificity G4-DNA stabilizing ligand, an ellipticine analogue GQC-05 (NSC338258) (Fig. 2d-f,j-1) ${ }^{16}$. In cells that were not treated with GNRs, we observed an increase (MCF-7, +104\%; HEK-293T, $+31.2 \%$ ) in the number of G4 foci following GQC-05 incubation (Fig. 2d,j,n-o), consistent with a previous study involving pyridostatin, an alternative G4 stabilizing ligand ${ }^{15}$. In cells with intracellular GNRs, GQC-05 resulted in an increase in the number of G4 foci back to normal levels (Fig. 2e,f,k,l,n,o). These results suggest 

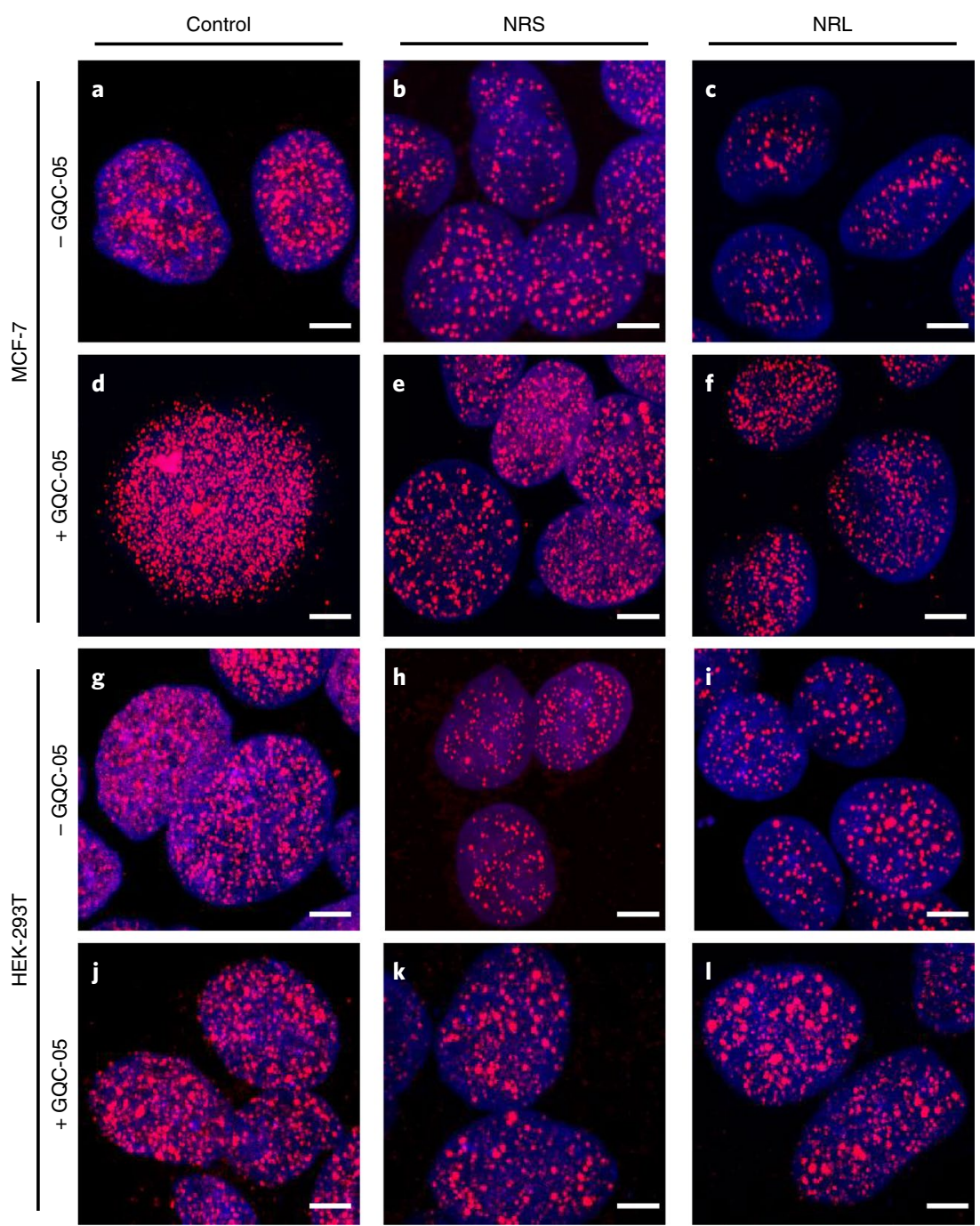

$\square$ Nuclei (DAPI)

G4 foci (Alexa 594)

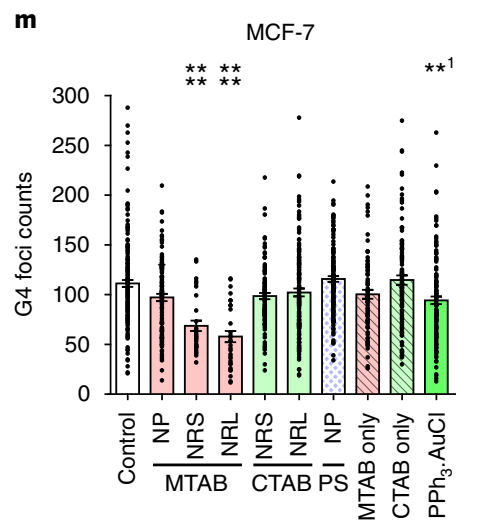

n

MCF-7

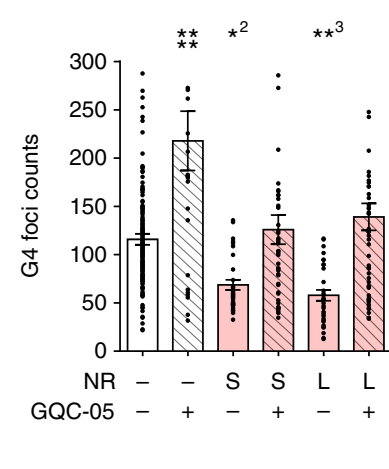

0

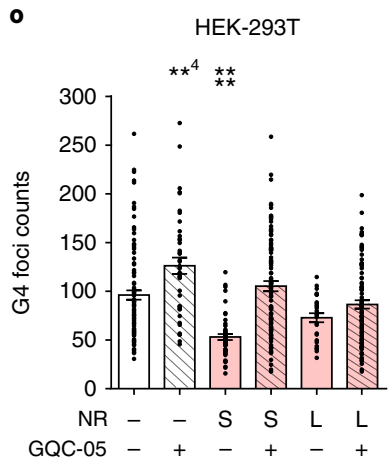

Fig. 2 | Alterations in G4-DNA formation in the genome, observed following gold nanorod internalization in cells. a-I, Visualization of G4 in MCF-7 (a-c) and HEK-293T ( $\mathbf{g}-\mathbf{i}$ ) cells treated with 0 or $100 \mu \mathrm{gl}^{-1}$ of NRS/NRL and without addition of the G4 stabilizing ligand GQC-05, and MCF-7 (d-f) and HEK$293 \mathrm{~T}(\mathbf{j}-\mathbf{I})$ cells treated with 0 or $100 \mu \mathrm{g} \mathrm{ml} \mathrm{I}^{-1}$ of NRS/NRL with the addition of GQC-05. a-I were performed three times independently, with similar results. $\mathbf{m}, \mathrm{G} 4$ foci counts in MCF-7 cells determined via confocal images. $\mathbf{n}, \mathbf{o}$, Comparison of G4 foci counts in NRS/NRL-treated cells, with and without GQC05 in MCF-7 (n) and HEK-293T (o) cells, respectively. One-way ANOVA was performed against control for $\mathbf{m}-\mathbf{0}$. ${ }^{\star * 1} P=0.003,{ }^{\star 2} P=0.015,{ }^{\star * 3} P=0.01$, ${ }^{\star \star 4} P=0.002,{ }^{\star \star \star \star} P<0.0001$. All data in graphs are presented as mean \pm s.e.m. and individual data points are shown as dots. Sample sizes for $\mathbf{m}-\mathbf{0}$ range from $n=25$ to 178 nuclei per condition from three biological replicates. Scale bars, $5 \mu$ m. NRS: AR 2.8 GNRs; NRL: AR 4.9 GNRs.

that the localization of GNRs within cytoplasmic vesicles can alter G4 formation, and the changes observed are dynamic and reversible in the presence of a G4 stabilizing ligand.
In thiol-stabilized gold nanoparticles, it has been established that the presence of $\mathrm{Au}(\mathrm{o})$-thiol species maintains the noble properties of gold, whereas the formation of $\mathrm{Au}(\mathrm{I})$-thiolates can disrupt this ${ }^{5}$. 

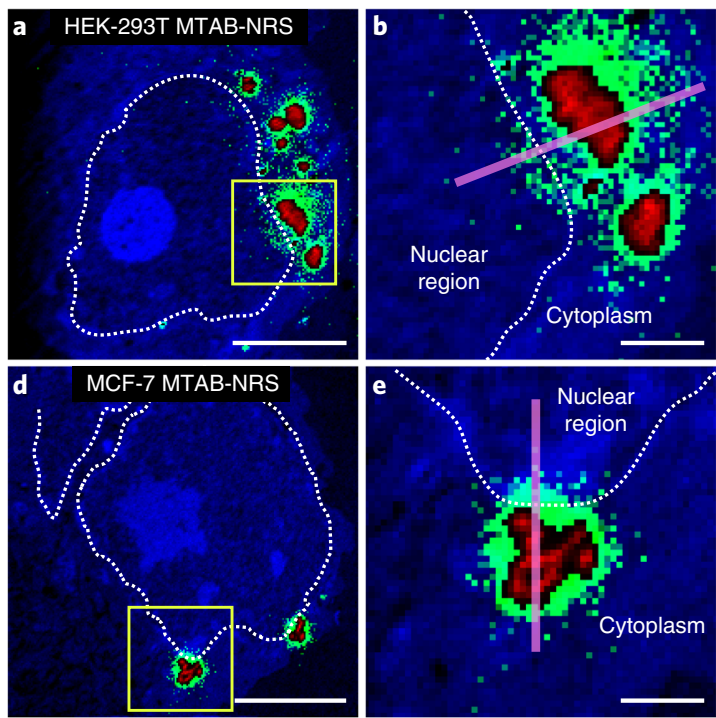

${ }^{12} \mathrm{C}^{14} \mathrm{~N}^{-}$elemental map

$\square{ }^{197} \mathrm{Au}^{-}$elemental map

NRS clusters
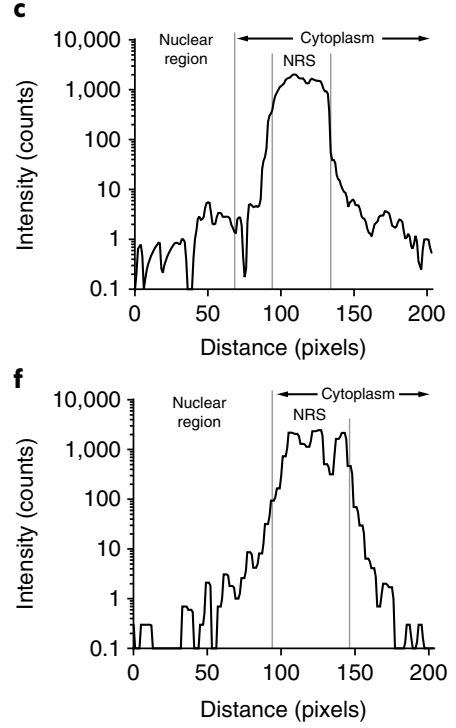

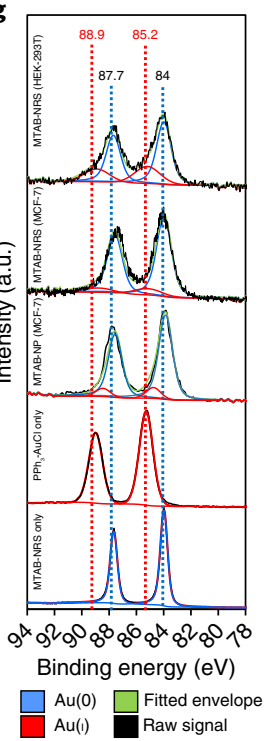

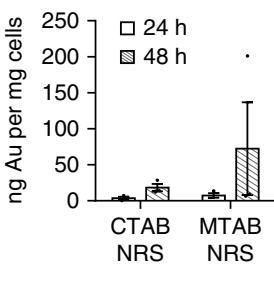

i

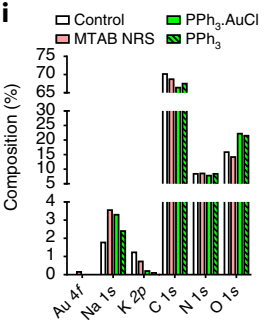

Fig. 3 I Intracellular chemical speciation of thiol-functionalized gold nanorods. a,b,d,e, Merged nanoscale secondary ion mass spectrometry elemental maps of representative HEK-293T (a,b) and MCF-7 (d,e) MTAB-NRS-treated samples. Magnified regions of interest in HEK-293T (a) and MCF-7 (d) cells, indicated by yellow boxes, are shown in $\mathbf{b}$ and $\mathbf{e}$, respectively. Au signals (green) can be observed within the nuclear region (dotted white line) away from the location of GNRs (in red). c,f, Representative intensity profiles of the diffused Au signals within the nuclei obtained from regions of interest represented by the pink lines in $\mathbf{b}$ and $\mathbf{e}$, respectively. $\mathbf{a}, \mathbf{b}, \mathbf{d}, \mathbf{e}$ were performed twice independently, with similar results. $\mathbf{g}$, X-ray photoelectron spectrum of the Au $4 f$ electron region of MTAB-NRS/NP-treated HEK-293T and MCF-7 cells and the corresponding spectra of PPh ${ }_{3}$.AuCl-only and NRS-only samples for reference. $\mathbf{h}$, Concentration of leached gold in cells after 24 and $48 \mathrm{~h}$ as assessed by ICP-MS ( $n=3$ per condition per time point). Data in $\mathbf{h}$ are presented as mean \pm s.e.m. and individual data points are shown as dots. i, Elemental composition of whole MCF-7 cells by proportion, as assessed by XPS. Scale bars, $5 \mu \mathrm{m}(\mathbf{a}, \mathbf{d}) ; 1 \mu \mathrm{m}(\mathbf{b}, \mathbf{e})$. NRS: AR $2.8 \mathrm{GNRs}$; NRL: AR 4.9 GNRs.

Given that $\mathrm{Au}(\mathrm{I})$-thiolates are less noble in their chemical properties, we queried if changes in oxidation states following intracellular localization can result in gold species leaching from the surface of GNRs into the nucleus, triggering the observed conformational changes of genomic DNA. This was validated by the $\mathrm{PPh}_{3}$. $\mathrm{AuCl}$ control treatment in MCF-7 cells also demonstrating a statistically significant decrease in G4 foci. To test this hypothesis, we employed nanoscale secondary ion mass spectrometry (NanoSIMS) imaging to monitor the distribution of gold species within HEK-293T and MCF-7 cells following cytoplasmic localization of AR 2.8 MTAB-GNRs. We observed the existence of a diffused gold signal outside cytoplasmic vesicles that were enriched with GNRs and, more importantly, the existence of gold species within the nucleus of both HEK-293T and MCF-7 cells with a corresponding Au ion signal within the nuclei ranging from 300 to 2,000 counts (Fig. 3a-f). In the case of MTABGNPs (Supplementary Fig. 5) we observed significant gold signals within the cytoplasmic vesicles with slightly diffused signals outside the vesicles, while no signals were detected within the nucleus. Conversely, with CTAB-GNRs (Supplementary Fig. 6), the viable cells indicated very low uptake of GNRs, with corresponding low signals in the cytoplasm and no signal in the nucleus. This further confirmed that significantly low uptake of CTAB-GNRs in the viable cells resulted in no significant changes in the G4 foci, as observed in Fig. 2m. For GNRs, growth and dissolution are spatioselective, preferentially occurring at the tips, with corresponding higher flux of ions and surfactants compared to GNPs ${ }^{17}$. In the present case, this could explain the higher intensity of diffused signals in the case of MTAB-GNRs when compared to GNPs. Representative elemental maps for both HEK-293T and MCF-7 cells are demonstrated in Supplementary Fig. 7. We next queried if the presence of these diffused gold signals in the nucleus of the MTAB-GNRs samples was associated with changes in the oxidation states of $\mathrm{Au}$, using X-ray photoelectron spectroscopy (XPS) (Fig. 3g). XPS analyses revealed that only the $\mathrm{Au}(\mathrm{o})$ state was observed for native GNRs before exposure to cells (Fig. 3g). As evidenced by the deconvoluted Au $4 f$ spectra, the presence of two different chemical states of Au was observed in physiological conditions following exposure of GNRs to cell culture media and following internalization in cells. The major doublet with the Au $4 f_{7 / 2}$ spin-orbit split component centred at $84 \mathrm{eV}$ corresponds to $\mathrm{Au}(\mathrm{o})$, while the smaller doublet positioned $\sim 1.2 \mathrm{eV}$ higher in binding energy is consistent with the presence of $\mathrm{Au}(\mathrm{I})^{18}$. The presence of $\mathrm{Au}(\mathrm{I})$ was further confirmed using $\mathrm{PPh}_{3} \cdot \mathrm{AuCl}$ as a control both in XPS, with matching peaks corresponding to $\mathrm{Au}(\mathrm{I})$, and in NanoSIMS within the nucleus of cells (Supplementary Fig. 5d-f), with the matching images corresponding to diffused $\mathrm{Au}(\mathrm{I})$ signals. These findings were further substantiated by ICP-MS analysis, which revealed the higher propensity of MTAB-GNRs to leach gold following exposure to cells in comparison to CTAB-coated GNRs (Fig. $3 \mathrm{~h}$ ). XPS analysis of the cell lysate supernatant following exposure of MTAB-GNRs to cells revealed that the leached gold corresponded to the $\mathrm{Au}$ (III) oxidation state (Supplementary Fig. 8). This is expected, as $\mathrm{Au}(\mathrm{III})$ is the most stable oxidation state in an extracellular environment; however, intracellular redox conditions coupled with the presence of stabilizing biomolecules like glutathione results in the formation of the stable $\mathrm{Au}(\mathrm{I})$ oxidation state ${ }^{19}$. Finally, XPS analysis of whole cells (MCF-7) following exposure to GNRs demonstrated that the introduction of nuclear localization $(\mathrm{Au}(\mathrm{I}))$ within the cells indeed deregulates the cellular electrophysiology, as evidenced by the measurable fluctuation in sodium $\left(\mathrm{Na}^{+}\right)$concentration within the cells (Fig. 3i), as compared to untreated control. This is important, as it is now widely reported that local changes in concentrations of $\mathrm{Na}^{+}$ and $\mathrm{K}^{+}$can directly impact the stability of G4-DNA structures ${ }^{20}$. We further substantiated these finding by measuring similar changes on exposure to $\mathrm{PPh}_{3} \cdot \mathrm{AuCl}$ and $\mathrm{PPh}_{3}$-only. We found that the measurable fluctuation in $\mathrm{Na}^{+}$concentration was indeed higher for the $\mathrm{PPh}_{3}$. $\mathrm{AuCl}$ condition. 

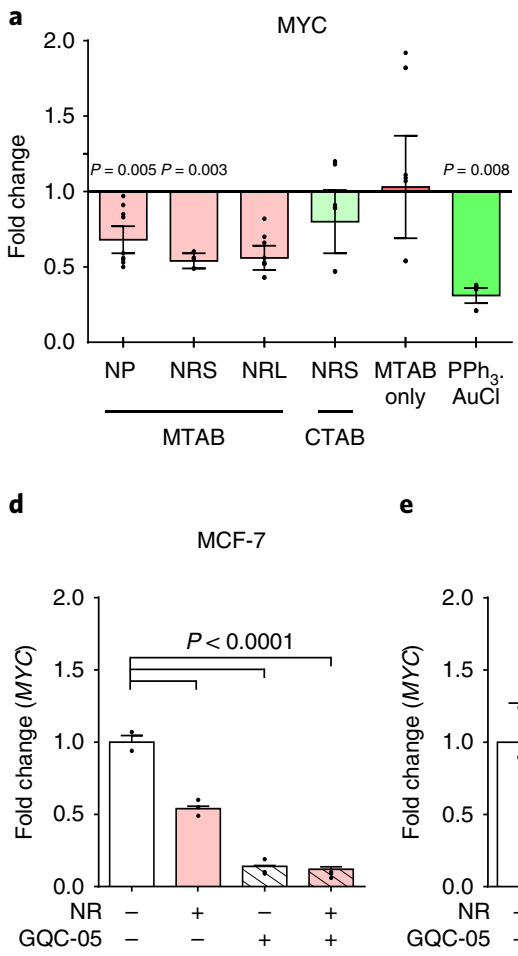

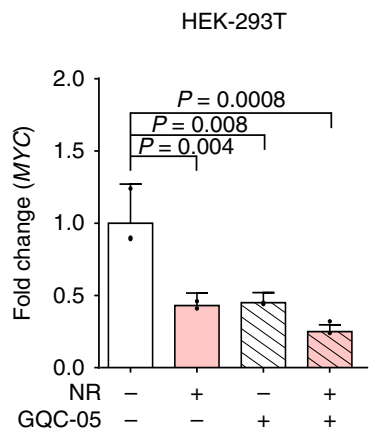

b

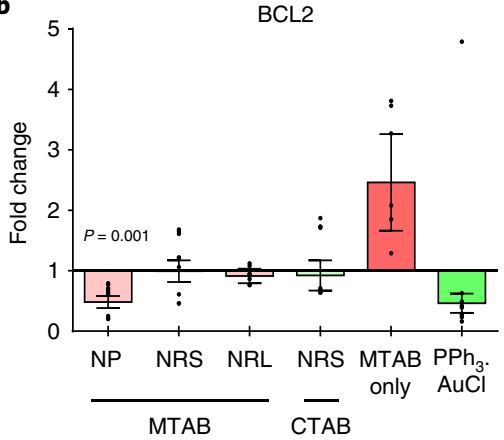

f

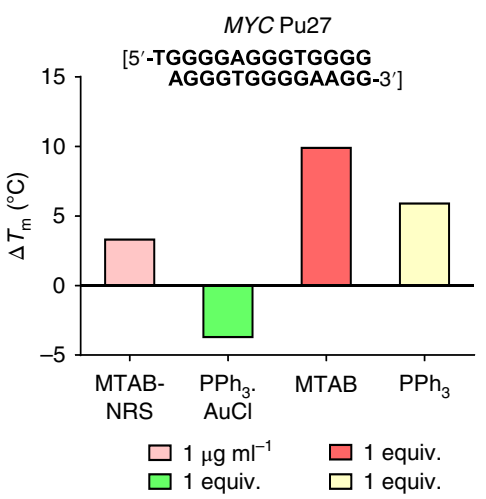

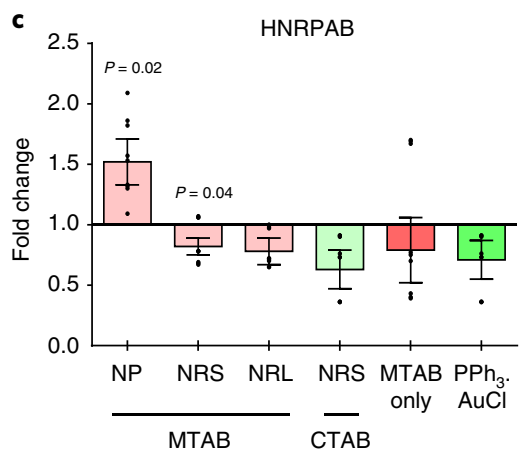

g

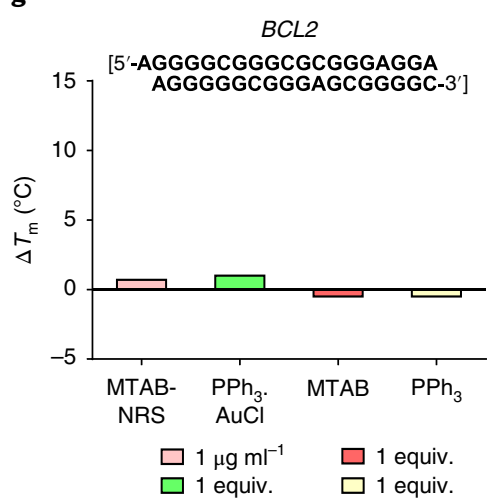

Fig. 4 | Alterations in gene expression associated with changes in $\mathbf{G} 4$ formation and gold nanorod internalization. a-c, Expression of $M Y C$ (a), $B C L 2$ (b) and HNRPAB (c) in treated MCF-7 cells as determined by GPCR ( $n=9$ per condition). All treated conditions were exposed to a dose of $100 \mu \mathrm{g} \mathrm{ml}^{-1}$ of the indicated condition over $48 \mathrm{~h}$, except for MTAB-only and $\mathrm{PPh}_{3} . \mathrm{AuCl}$, which were administered at their respective $\mathrm{IC}_{50}$ dose over $48 \mathrm{~h}$. $\mathbf{d}$,e, Expression of MYC in MCF-7 (d) and HEK-293T (e) cells, as determined by qPCR ( $n=3$ per condition). Conditions with $+\mathrm{GNR}$ were treated with $100 \mu \mathrm{g} \mathrm{ml}^{-1}$ of NRS in the presence and absence of GQC-05. Fold changes in expression were compared against control (-GNR, $-\mathrm{GQC}-05)$ cells. $\mathbf{f}, \mathbf{g}$, Difference in melting temperature $\left(\Delta T_{\mathrm{m}}\right)$ of $M Y C$-forming (f) and BCL2 G4-forming (g) oligomers when exposed to 1 molar equivalent (or $1 \mu \mathrm{g} \mathrm{ml}{ }^{-1}$ for MTAB-NRS) of selected compounds compared to controls. One-way ANOVA was performed against control for a-e. All data in graphs are presented as mean \pm s.e.m. and individual data points are shown as dots. NRS: AR 2.8 GNRs; NRL: AR 4.9 GNRs.

We next evaluated whether these observed changes in G4 foci following GNR exposure can be associated with changes in gene expression. For this purpose, we chose to evaluate changes in $M Y C, B C L 2$ and HNRPAB gene expression, as these have high, intermediate and low potential to form G4 structures within the genome, respectively. We monitored the changes in these respective genes following exposure to MTAB-GNRs/GNPs with appropriate controls (Fig. $4 \mathrm{a}-\mathrm{c}$ ). In the case of $M Y C$ we observed a statistically significant downregulation of $M Y C$ mRNA expression following treatment with MTAB-coated GNRs and GNPs (Fig. 4a). Additionally, we observed a statistically significant downregulation of $M Y C$ expression following exposure to $\mathrm{PPh}_{3} \cdot \mathrm{AuCl}$, demonstrating the potential contribution of $\mathrm{Au}(\mathrm{I})$ species in changes to gene expression (Fig. 4a). This is further supported by the NanoSIMS images, where Au signals were observed in the nuclei of $\mathrm{PPh}_{3}$. AuCltreated samples (Supplementary Fig. 5d-f). For BCL2, statistically significant changes were only observed for MTAB-GNPs (Fig. 4b). Significant changes in expression of $H N R P A B$ were observed for both MTAB-GNRs and GNPs; this was surprising given the low G4 formation potential obtained for this gene (Fig. 4c). It is therefore pivotal to highlight that changes in gene expression can be attributed to alterations in a highly complex process in which several regulatory factors may independently and perhaps simultaneously operate at various stages of transcription and translation of the gene. For instance, intracellular accumulation of GNPs at low (non-toxic) doses can result in elevated cellular stress, which in turn can significantly alter both $B C L 2$ and $H N R P A B$ gene expressions ${ }^{21,22}$. This can explain additional contributors to the observed changes in BCL2 and $H N R P A B$ following MTAB-GNPs exposure. Furthermore, in the case of $M Y C$ we also used the G4 interacting ligand GQC-05 as a control as it has been shown to bind to the G4 structure in the nuclease hypersensitive element $\mathrm{III}_{1}\left(\mathrm{NHE} \mathrm{III}_{1}\right)$ region of $M Y C$ to downregulate transcription of $M Y C \mathrm{mRNA}^{16}$. In the case of $M Y C$, we observed a statistically significant downregulation of $M Y C$ expression following treatment with MTAB-GNRs and GQC-05 at a concentration below observed $\mathrm{IC}_{50}$ values (Supplementary Fig. 9), both separately and in combination (Fig. 4d,e). Taken together, although accounting for singular contributions for changes in gene expression following GNR and GNP exposure may not be prudent, we believe that our study highlights that the speciation of intracellular GNRs (and the concomitant structural alternations in genomic DNA) is a very important and previously overlooked factor. To highlight this further, we performed circular dichroism (CD) melt experiments using synthetic oligos that mimic the MYC and BCL2 quadruplexes (Fig. 4f,g) ${ }^{23,24}$. An increase in the melting temperature $T_{\mathrm{m}}$ of the oligos is indicative of stabilization, and a decrease indicates disruption of the G4 structures. We observed a decrease in $T_{\mathrm{m}}$ in the case of $\mathrm{PPh}_{3}$. AuCl and an increase for the rest, especially $\mathrm{PPh}_{3}$, in the absence of $\mathrm{Au}(\mathrm{I})$ for $M Y C$, and no changes in $T_{\mathrm{m}}$ for all samples in the case of BCL2. The observed increase in melt temperatures in the presence of colloids and surfactant is attributed to the previously observed phenomenon of G4 structural stabilization by the molecular crowding effect ${ }^{25}$. This experiment highlights two important aspects: that the presence of $\mathrm{Au}(\mathrm{I})$ species does indeed have 
the potential to induce structural changes in G4-DNA, and that its presence does not universally alter G4-DNA structures across the whole genome. This finding is similar to that observed for several small-molecule G4 stabilizing ligands, which show differential stabilization potential for various genes ${ }^{16,26}$.

In summary, we have demonstrated that the ubiquitous thiol chemistry used to engineer GNRs for intracellular applications in drug and gene delivery can alter the microenvironment within the nucleus as a result of leached gold species. The study has focused on the conformational dynamics of genomic DNA using G4 structures as a marker, and we surmise that nuclear localization of gold in the present case may also affect the interactions and dynamics of other regulatory molecules (including transcription factors and histone proteins) with genomic DNA as a result of changes in the chemical microenvironment. This study demonstrates that to develop translatable technologies using gold nanomaterials, it is essential to explore in situ chemical changes within the genomic environment in detail, beyond the traditional coarse cytotoxicity evaluation.

\section{Online content}

Any methods, additional references, Nature Research reporting summaries, source data, statements of data availability and associated accession codes are available at https://doi.org/10.1038/ s41565-018-0272-2.

Received: 12 February 2018; Accepted: 3 September 2018; Published online: 08 October 2018

\section{References}

1. Mieszawska, A. J., Mulder, W. J. M., Fayad, Z. A. \& Cormode, D. P. Multifunctional gold nanoparticles for diagnosis and therapy of disease. Mol. Pharm. 10, 831-847 (2013).

2. Chithrani, B. D., Ghazani, A. A. \& Chan, W. C. W. Determining the size and shape dependence of gold nanoparticle uptake into mammalian cells. Nano Lett. 6, 662-668 (2006).

3. Qiu, Y. et al. Surface chemistry and aspect ratio mediated cellular uptake of Au nanorods. Biomaterials 31, 7606-7619 (2010).

4. Hauck, T. S., Ghazani, A. A. \& Chan, W. C. W. Assessing the effect of surface chemistry on gold nanorod uptake, toxicity, and gene expression in mammalian cells. Small 4, 153-159 (2008).

5. Reimers, J. R., Ford, M. J., Halder, A., Ulstrup, J. \& Hush, N. S. Gold surfaces and nanoparticles are protected by $\mathrm{Au}(\mathrm{o})$-thiyl species and are destroyed when Au(I)-thiolates form. Proc. Natl Acad. Sci. USA 113, E1424-E1433 (2016).

6. Vigderman, L., Manna, P. \& Zubarev, E. R. Quantitative replacement of cetyl trimethylammonium bromide by cationic thiol ligands on the surface of gold nanorods and their extremely large uptake by cancer cells. Angew. Chem. Int. Ed. 51, 636-641 (2012)

7. Alkilany, A. M. et al. Cellular uptake and cytotoxicity of gold nanorods: molecular origin of cytotoxicity and surface effects. Small 5, 701-708 (2009).

8. Wang, L. et al. Surface chemistry of gold nanorods: origin of cell membrane damage and cytotoxicity. Nanoscale 5, 8384 (2013).

9. Railsback, J. G. et al. Weakly charged cationic nanoparticles induce DNA bending and strand separation. Adv. Mater. 24, 4261-4265 (2012).

10. Lankaš, F., Šponer, J., Hobza, P. \& Langowski, J. Sequence-dependent elastic properties of DNA. J. Mol. Biol. 299, 695-709 (2000).

11. Packer, M. J., Dauncey, M. P. \& Hunter, C. A. Sequence-dependent DNA structure: tetranucleotide conformational maps. J. Mol. Biol. 295, 85-103 (2000).

12. Bochman, M. L., Paeschke, K. \& Zakian, V. A. DNA secondary structures: stability and function of G-quadruplex structures. Nat. Rev. Genet. 13, 770-780 (2012).

13. Lam, E. Y. N., Beraldi, D., Tannahill, D. \& Balasubramanian, S. G-quadruplex structures are stable and detectable in human genomic DNA. Nat. Commun. 4, 1796 (2013)

14. David, A. P. et al. G-quadruplexes as novel cis-elements controlling transcription during embryonic development. Nucleic Acids Res. 44 4163-4173 (2016).
15. Biffi, G., Tannahill, D., McCafferty, J. \& Balasubramanian, S. Quantitative visualization of DNA G-quadruplex structures in human cells. Nat. Chem. 5, 182-186 (2013).

16. Brown, R. V., Danford, F. L., Gokhale, V., Hurley, L. H. \& Brooks, T. A. Demonstration that drug-targeted down-regulation of MYC in non-Hodgkins lymphoma is directly mediated through the promoter G-quadruplex. J. Biol. Chem. 286, 41018-41027 (2011).

17. Rodríguez-Fernández, J., Pérez-Juste, J., Mulvaney, P. \& Liz-Marzán, L. M. Spatially-directed oxidation of gold nanoparticles by Au(III)-CTAB complexes. J. Phys. Chem. B 109, 14257-14261 (2005).

18. Casaletto, M. P., Longo, A., Martorana, A., Prestianni, A. \& Venezia, A. M. XPS study of supported gold catalysts: the role of $\mathrm{Au} 0$ and $\mathrm{Au}+\delta$ species as active sites. Surf. Interface Anal. 38, 215-218 (2006).

19. Zou, T., Lum, C. T., Lok, C.-N., Zhang, J.-J. \& Che, C.-M. Chemical biology of anticancer gold(III) and gold(I) complexes. Chem. Soc. Rev. 44, 8786-8801 (2015).

20. Campbell, N. H. \& Neidle, S. in Interplay between Metal Ions and Nucleic Acids (eds Sigel, A., Sigel, H. \& Sigel, R. K. O.) 119-134 (Springer, Dordrecht, 2012).

21. Gunduz, N., Ceylan, H., Guler, M. O. \& Tekinay, A. B. Intracellular accumulation of gold nanoparticles leads to inhibition of macropinocytosis to reduce the endoplasmic reticulum stress. Sci. Rep. 7, 40493 (2017).

22. Sinnamon, J. R., Waddell, C. B., Nik, S., Chen, E. I. \& Czaplinski, K. Hnrpab regulates neural development and neuron cell survival after glutamate stimulation. RNA 18, 704-719 (2012).

23. Dai, J., Chen, D., Jones, R. A., Hurley, L. H. \& Yang, D. NMR solution structure of the major G-quadruplex structure formed in the human BCL2 promoter region. Nucleic Acids Res. 34, 5133-5144 (2006).

24. Siddiqui-Jain, A., Grand, C. L., Bearss, D. J. \& Hurley, L. H. Direct evidence for a G-quadruplex in a promoter region and its targeting with a small molecule to repress c-MYC transcription. Proc. Natl Acad. Sci. USA 99, 11593-11598 (2002).

25. Zheng, K., Chen, Z., Hao, Y. \& Tan, Z. Molecular crowding creates an essential environment for the formation of stable G-quadruplexes in long double-stranded DNA. Nucleic Acids Res. 38, 327-338 (2010).

26. Neidle, S. Quadruplex nucleic acids as novel therapeutic targets. J. Med. Chem. 59, 5987-6011 (2016).

\section{Acknowledgements}

The authors thank S. Balasubramanian for his gift of pSANG10-3F-BG4. This research was supported by the Australian Research Council, the National Health and Medical Research Council and the Raine Medical Research Foundation for the Healy Research Collaboration Award. The authors acknowledge the facilities and the scientific and technical assistance of the Australian Microscopy \& Microanalysis Research Facility at the Centre for Microscopy, Characterisation \& Analysis, The University of Western Australia, a facility funded by the University, State and Commonwealth Governments, as well as the WA X-ray Surface Analysis Facility of the John de Laeter Centre, funded by the Australian Research Council LIEF grant LE120100026. J.A.Kr. acknowledges Cancer Council Western Australia for a PhD Top Up scholarship.

\section{Author contributions}

N.M.S. and K.S.I. developed the hypothesis. D.H., J.A.Ke. and K.S.I designed the GNR intracellular evaluation experiments. N.M.S., L.H.H., J.A.Kr., D.H. and R.C. designed the G4 interaction experiments. C.W.E., D.H. and J.A.Kr. performed the cell viability and toxicity analysis. M.No. performed the MTAB synthesis. N.M.S., M.Ng., A.L.K., A.J.B. and C.S.B. carried out BG4 antibody production and purification. T.D.C., J.A.Kr. and J.-P.V. performed the XPS experiments. D.H., J.A.Kr., P.G., H.J. and M.R.K. performed the NanoSIMS experiments. D.H. and P.T. performed the qPCR experiments. A.M.M., M.S. and D.H. performed the nanoparticle synthesis and characterization. J.A.Kr. and C.W.E. performed ICP experiments. M.A. and M.F. performed TEM/NanoSIMS sample preparation and analysis. All authors contributed to writing the manuscript.

\section{Competing interests}

The authors declare no competing interests.

\section{Additional information}

Supplementary information is available for this paper at https://doi.org/10.1038/ s41565-018-0272-2.

Reprints and permissions information is available at www.nature.com/reprints. Correspondence and requests for materials should be addressed to N.M.S. or K.S.I. Publisher's note: Springer Nature remains neutral with regard to jurisdictional claims in published maps and institutional affiliations.

(c) The Author(s), under exclusive licence to Springer Nature Limited 2018 


\section{Methods}

Materials. All chemicals were purchased from Sigma-Aldrich unless otherwise stated: (CTAB; $\geq 98 \%)$, hydrogen tetrachloroaurate(III) hydrate $\left(\mathrm{HAuCl}_{4} ; 99.9 \%\right.$, Alfa Aesar), trisodium citrate ( $99 \%$, Ajax Finechem), sodium oleate ( $>97 \%$, Tokyo Chemical Industry), $\mathrm{AgNO}_{3}$ (99\%, Chem-Supply), polystyrene nanospheres (25 $\mathrm{nm}$ average diameter, $\mathrm{Phosphorex)}$. $\mathrm{PPh}_{3}$. AuCl was purchased as a control from Sigma-Aldrich at $\geq 99.9 \%$ purity. All glassware was cleaned with fresh aqua regia followed by rinsing with ultrapure water before being used for gold nanorod syntheses. pSANG10-3F-BG4 plasmid encoding for BG4 antibody was a gift from S. Balasubramanian (Addgene plasmid $\# 55756)^{15}$.

MTAB synthesis. MTAB was synthesized as previously reported ${ }^{6}$. Briefly, to a stirred solution of 16-bromo-1-hexadecanethiol $(0.85 \mathrm{mmol})$ in ethyl acetate $(5 \mathrm{ml})$, a $4.2 \mathrm{M}$ ethanolic solution of trimethylamine $(3 \mathrm{ml})$ was added and the resulting mixture was stirred under argon for 4 days. A white precipitate was filtered and purified by several washes with ethyl acetate to remove excess trimethylamine. The product was subsequently lyophilized and stored in a desiccator.

GNP and GNR syntheses and characterization. GNPs and GNRs were synthesized using seed-mediated approaches, as described previously ${ }^{27,28}$.

GNP synthesis. To prepare the gold seed solution for GNPs, $0.6 \mathrm{ml}$ of ice-cold $0.1 \mathrm{M}$ $\mathrm{NaBH}_{4}$ was added to a $20 \mathrm{ml}$ solution of $0.25 \mathrm{mM}$ trisodium citrate and $0.25 \mathrm{mM}$ $\mathrm{HAuCl}_{4}$ under vigorous stirring.

CTAB ( $80 \mathrm{mM}, 2.1 \mathrm{~g}$ ) was dissolved in in $72 \mathrm{ml}$ of $2.5 \mathrm{mM} \mathrm{HAuCl}_{4}$ to prepare growth solution. The growth solution was heated to $50^{\circ} \mathrm{C}$ to aid dissolution of $\mathrm{CTAB}$ and then cooled to $30^{\circ} \mathrm{C}$ before use.

GNPs $(19 \mathrm{~nm})$ were synthesized in two growth stages from the initial gold seed solution. In the first growth stage, $9 \mathrm{ml}$ of growth solution was reduced by $0.5 \mathrm{ml}$ of ascorbic acid $(0.1 \mathrm{M})$. To this solution, $10 \mathrm{ml}$ of gold seed solution was added rapidly under vigorous stirring. The solution was left to stir at the same speed for $10 \mathrm{~min}$. Thereafter, the suspension (Set A) was aged for a further $30 \mathrm{~min}$ at room temperature before use.

In the second growth stage, a separate solution containing $57.5 \mathrm{ml}$ of growth solution was reduced by $2.5 \mathrm{ml}$ of ascorbic acid $(0.1 \mathrm{M})$. A $5 \mathrm{ml}$ volume of Set A was added rapidly to this solution under vigorous stirring. The suspension was left stirring for $10 \mathrm{~min}$ before collection and centrifugation at $24,000 \mathrm{~g}$ for $30 \mathrm{~min}$ to obtain $19 \mathrm{~nm}$ GNPs.

GNR synthesis. To prepare the gold seed solution for the GNR, $0.6 \mathrm{ml}$ of ice-cold $0.01 \mathrm{M} \mathrm{NaBH}_{4}$ was injected into a solution composed of $5 \mathrm{ml} \mathrm{HAuCl}_{4}(0.5 \mathrm{mM})$ and $5 \mathrm{ml} \mathrm{CTAB}(0.2 \mathrm{M})$ under vigorous stirring for $2 \mathrm{~min}$. The seed solution was aged for $30 \mathrm{~min}$ at room temperature before being used in the synthesis of NRS (AR 2.8 GNRs; $54.6 \mathrm{~nm} \times 19 \mathrm{~nm}$ ) and NRL (AR 4.9 GNRs; $91.5 \mathrm{~nm} \times 19 \mathrm{~nm}$ ).

The growth solution was prepared with $0.62 \mathrm{~g}$ sodium oleate and either $4.5 \mathrm{~g}$ (for NRS) or $3.5 \mathrm{~g}$ (for NRL) CTAB in $125 \mathrm{ml}$ of water. The mixture was warmed to $50^{\circ} \mathrm{C}$ to aid dissolution of sodium oleate and $\mathrm{CTAB}$, and cooled to $30^{\circ} \mathrm{C}$ before use. The growth solution was then maintained at $30^{\circ} \mathrm{C}$ for all subsequent steps in the synthesis. A $6 \mathrm{ml}$ volume (for GNRs) or $12 \mathrm{ml}$ (for GNRL) of $4 \mathrm{mM} \mathrm{AgNO}$ was added to the solution and kept undisturbed for $15 \mathrm{~min}$. Next, $125 \mathrm{ml} \mathrm{HAuCl}$ $(1 \mathrm{mM})$ was added to the solution with moderate stirring for $90 \mathrm{~min}$. After this, $1.3 \mathrm{ml}$ (for NRS) or $3.2 \mathrm{ml}$ (for NRL) of $\mathrm{HCl}(32 \mathrm{wt} \%, 10.2 \mathrm{M}$ ) was added to the colourless growth solution and kept for $15 \mathrm{~min}$ under slow stirring. Ascorbic acid $(64 \mathrm{mM}, 0.625 \mathrm{ml})$ was added with vigorous stirring for $30 \mathrm{~s}$. Finally, $0.4 \mathrm{ml}$ of the seed solution was added to the growth solution, vigorously stirred for $30 \mathrm{~s}$ and then left undisturbed at $30^{\circ} \mathrm{C}$ for $12 \mathrm{~h}$ to allow nanorod growth. The suspensions were collected and centrifuged at $24,000 \mathrm{~g}$ for $30 \mathrm{~min}$ to obtain GNRs.

CTAB to MTAB ligand exchange. The CTAB surface coating on the GNPs/ GNRs was exchanged with MTAB in water in a 1:1 ratio by mass over $48 \mathrm{~h}$ under moderate stirring. Thereafter, the GNP/GNR suspensions were centrifuged at $24,000 \mathrm{~g}$ for $30 \mathrm{~min}$ and the supernatant containing CTAB was removed. Ultrapure water was used to resuspend the GNPs/GNRs, and this process was repeated six times in total. The final concentration of GNPs/GNRs was determined by ICP-MS. These MTAB-coated GNPs/GNRs were used in all subsequent experiments.

The MTAB-CTAB exchange on the surface of the GNPs/GNRs was analysed with EELS on a Gatan Enfinium spectrometer on a FEI Titan G2 80-200 TEM/ STEM operating at $200 \mathrm{kV}$. All spectra were acquired using a dispersion of $0.1 \mathrm{eV}$ per pixel and an acquisition time of $1 \mathrm{~s}$, with each spectrum being the sum of ten acquisitions to improve the signal-to-noise ratio. The multiple scattering background was removed by fitting the data in the energy range before the typical energy onset for the $\mathrm{S} L_{2,3}$ edge (at $\sim 160 \mathrm{eV}$ energy loss).

Cell culture and treatment with gold nanomaterials. Human embryonic kidney cells (HEK-293T, ATCC, confirmed to be mycoplasma negative) and human breast adenocarcinoma cells (MCF-7, ATCC, confirmed to be mycoplasma negative) were cultured in T75 cell culture treated flasks with filter caps (ThermoFisher Scientific) in a humidified atmosphere containing $5 \% \mathrm{CO}_{2}$ at $37^{\circ} \mathrm{C}$, and maintained in DMEM (for HEK-293T) or MEM- $\alpha$ (for MCF-7) further supplemented with fetal bovine serum (10\% vol/vol) and GlutaMAX (ThermoFisher Scientific).
HEK-293T and MCF-7 cells were incubated for $24 \mathrm{~h}$ in six-well plates before the cell medium was replaced with GNP/GNR suspensions $\left(0,10\right.$ or $100 \mu \mathrm{g} \mathrm{ml}^{-1}$ final concentration of GNPs/GNRs) made up in fresh cell medium. The cells were further incubated at $5 \% \mathrm{CO}_{2}, 37^{\circ} \mathrm{C}$ for $48 \mathrm{~h}$ before being used for further analysis.

Cell viability assays. The cell viability of HEK-293T and MCF-7 cells treated with GNPs/GNRs were measured using a LIVE/DEAD cytotoxicity kit (ThermoFisher Scientific). The assay was performed according to the manufacturer's protocol and imaged on an inverted fluorescence microscope (Olympus IX71). Three images were recorded from each well $(n=3$ per condition) at consistent locations for all wells at $\times 10$ magnification. Mean cell viability was represented as the proportion of live cells over the total cell count (both live and dead) and normalized to the control condition $\left(0 \mu \mathrm{g} \mathrm{ml} \mathrm{m}^{-1}\right.$ nanorod concentration) for each time point.

Cytotoxicity assays of GQC-05 ( $1 \mathrm{nM}$ to $1 \mathrm{mM}), \mathrm{CTAB}(50 \mathrm{pM}$ to $5 \mathrm{mM})$ and MTAB (50 pM to $0.5 \mathrm{mM}$ ) in HEK-293T and MCF-7 cells were performed using an MTS assay (Promega). The $\mathrm{IC}_{50}$ values of GQC-05, CTAB and MTAB in HEK293T and MCF-7 cells were determined using a nonlinear regression model with GraphPad Prism statistical analysis software (GraphPad Software).

ICP-MS. Rate of GNR uptake into cells. MCF-7 cells were seeded overnight at a density of $2.5 \times 10^{5}$ cells per well in six-well plates, and treated with GNRs with either MTAB or CTAB surface coating $\left(100 \mu \mathrm{g} \mathrm{ml}^{-1}\right.$ made up in fresh cell medium) for a further 24 and $48 \mathrm{~h}$. At the designated time point, cells were washed thoroughly with PBS, trypsinized and counted. Cells were centrifuged $(200 \mathrm{~g}$ for $5 \mathrm{~min}$ ) and the pellet washed with PBS before being centrifuged again and the supernatant carefully removed. Cells were resuspended in MilliQ water $(200 \mu \mathrm{l})$ and transferred to a digestion vessel where freshly made aqua regia $(800 \mu \mathrm{l})$ was added. Samples were incubated at room temperature $(1 \mathrm{~h})$ before MilliQ water $(4 \mathrm{ml})$ was added. Conditions were tested in triplicate, with untreated cells $(n=3)$ and MilliQ water $(n=3)$ as a control and blank respectively. Samples were analysed by ICP-MS, conducted by TSW Analytical.

Rate of intracellular Au leaching from GNR. MCF-7 cells were seeded overnight at a density of $2.5 \times 10^{5}$ cells per well in six-well plates, and treated with GNRs with either MTAB or CTAB surface coating $\left(100 \mu \mathrm{g} \mathrm{ml}^{-1}\right.$ made up in fresh cell medium) for a further 24 and $48 \mathrm{~h}$. At the designated time point, cells were washed thoroughly with PBS, trypsinized and counted. Cells were centrifuged ( $200 g$ for $5 \mathrm{~min}$ ) and the pellet washed with PBS before being centrifuged again and the supernatant carefully removed. Cells were resuspended in MilliQ $(500 \mu \mathrm{l})$ and sonicated until samples were homogenized (4 W, 10s). Samples were centrifuged $(16,000 \mathrm{~g}, 10 \mathrm{~min})$ to pellet GNRs and cell debris, before supernatant $(450 \mu \mathrm{l})$ was carefully transferred to a digestion vessel for analysis. Samples were then diluted to a total of $5 \mathrm{ml}$ in a fresh solution of $1 \% \mathrm{HCl}$ made up in MilliQ. Conditions were tested in triplicate, with untreated cells $(n=3)$ and MilliQ water $(n=3)$ as a control and blank, respectively. Samples were analysed by ICP-MS, conducted by TSW Analytical.

BG4 antibody synthesis. BL21 (DE3) Escherichia coli cells were transformed with pSANG10-3F-BG4 plasmid, plated on antibiotic-treated agar (kanamycin, $50 \mu \mathrm{g} \mathrm{ml}^{-1}$ ) and incubated overnight at $37^{\circ} \mathrm{C}$. Thereafter, six single cell colonies were picked and used to inoculate six tubes containing $5 \mathrm{ml}$ of kanamycintreated Luria-Bertani (LB) medium. Pre-cultures were stored in an orbital shaker overnight at $37^{\circ} \mathrm{C}$. Each tube of pre-culture was used to inoculate conical flasks containing $500 \mathrm{ml}$ kanamycin-treated $2 \mathrm{YT}$ media supplemented with $1 \%$ $\mathrm{wt} / \mathrm{vol} \mathrm{D}$-glucose. Main cultures were incubated at $37^{\circ} \mathrm{C}$ in an orbital shaker until $\Delta \mathrm{OD}_{600 \mathrm{~nm}}=0.6$, to ensure that cells remained in an exponential growth phase. Once cultures reached the desired $\Delta \mathrm{OD}_{600 \mathrm{~nm}}$, they were induced with $1 \mathrm{mM}$ isopropyl $\beta$-D-1-thiogalactopyranoside (IPTG) and incubated overnight in an orbital shaker at $25^{\circ} \mathrm{C}$. Following incubation, main cultures were centrifuged at $4,000 \mathrm{~g}$ for $45 \mathrm{~min}$ at $4^{\circ} \mathrm{C}$, following which the pellet was resuspended in $2 \mathrm{YT}$ medium. The resuspended solution was then centrifuged at $4,000 \mathrm{~g}$ for $45 \mathrm{~min}$ at $4{ }^{\circ} \mathrm{C}$ until a pellet formed. Cell pellets were resuspended in $40 \mathrm{ml}$ lysis buffer ( $50 \mathrm{mM}$ Tris, $1 \mathrm{M} \mathrm{KCl}, 20 \mathrm{mM}$ imidazole, $\mathrm{pH} 8.0$ ). DNase was added to each tube before the bacteria were lysed under high pressure (Emulsiflex C5 homogenizer, Avestin). Each solution was passed through three times to achieve adequate lysis and kept on ice between each pass. Following lysis, samples were centrifuged at $20,000 \mathrm{~g}$ for $40 \mathrm{~min}$ at $4^{\circ} \mathrm{C}$. The supernatant was then loaded onto a $1 \mathrm{ml}$ HisTrap HP column (GE Healthcare). The column was washed with lysis buffer and followed by elution of BG4 in an elution buffer $(50 \mathrm{mM}$ Tris, $250 \mathrm{mM} \mathrm{KCl}$, $500 \mathrm{mM}$ imidazole, $\mathrm{pH} 8.0$ ). The eluant was further purified via size exclusion chromatography using a HiLoad 16/60 Superdex 200 prep grade column (GE Healthcare) on a BioLogic DuoFlow chromatography system with a QuadTec detector (Bio-Rad). Purified BG4 was eluted with size exclusion buffer ( $50 \mathrm{mM}$ Tris, $250 \mathrm{mM} \mathrm{KCl}, \mathrm{pH} 8.0$ ). The presence of purified BG4 in the eluant was confirmed by $12 \%$ SDS-PAGE (Mini-Protean TGX Stain-Free gel, Bio-Rad) with stain-free imaging on a ChemiDoc MP system (Bio-Rad). The concentration of BG4 was determined spectrophotometrically at $280 \mathrm{~nm}$ using a NanoDrop Lite spectrophotometer (ThermoFisher Scientific). 
Confocal microscopy. HEK-293T and MCF-7 cells were seeded at 1.5 and $2.5 \times 10^{5}$ cells per well, respectively, in six-well plates on top of poly-L-lysine-coated glass coverslips (ProSciTech) overnight, before incubation with GNRs/L suspensions $\left(0,10\right.$ or $100 \mu \mathrm{g} \mathrm{ml}^{-1}$ final concentration of nanorods), CTAB $\left(5 \mu \mathrm{g} \mathrm{ml}^{-1}\right)$, MTAB $\left(5 \mu \mathrm{g} \mathrm{ml}^{-1}\right)$ or $\mathrm{PPh}_{3} \cdot \mathrm{AuCl}\left(1.25 \mu \mathrm{g} \mathrm{ml}^{-1}\right)$ made up in fresh cell medium. The cells were further incubated at $5 \% \mathrm{CO}_{2}, 37^{\circ} \mathrm{C}$ for $48 \mathrm{~h}$. The samples were incubated with cytoplasm removal buffer ( $20 \mathrm{mM}$ HEPES-KOH, $20 \mathrm{mM} \mathrm{NaCl}, 5 \mathrm{mM} \mathrm{MgCl}$ $300 \mathrm{mM}$ sucrose, $0.5 \%$ (vol/vol) NP-40, $\mathrm{pH} 7.9$ ) for $20 \mathrm{~min}$ before being fixed in $2 \%$ paraformaldehyde/PBS followed by permeabilization with $0.1 \%$ Triton-X100/ PBS. Selected samples were then treated with $10 \mu \mathrm{M}$ of GQC- $05^{16}$ for $1 \mathrm{~h}$, while samples without GQC-05 were kept in PBS for the same time period. Thereafter, all samples were blocked with $2 \%$ skimmed milk/PBS and subjected to standard immunofluorescence methods with BG4, anti-FLAG (\#2368, Cell Signaling Technology) and anti-rabbit Alexa Fluor 594 (A-11037, ThermoFisher Scientific) antibodies. The coverslips were mounted with SlowFade Gold antifade reagent with DAPI (4',6-diamidino-2-phenylindole; ThermoFisher Scientific). Sections were imaged by confocal microscopy (Nikon A1Si, Centre for Microscopy, Characterization and Analysis, The University of Western Australia). DAPI was imaged at $\lambda_{\mathrm{ex}}=405 \mathrm{~nm}$ and $\lambda_{\mathrm{em}}=450 / 25 \mathrm{~nm}$, while Alexa Fluor 594 was imaged at $\lambda_{\mathrm{ex}}=561 \mathrm{~nm}$ and $\lambda_{\mathrm{em}}=595 / 25 \mathrm{~nm}$. Images were captured at $\times 120$ magnification, and the entirety of the cell nuclei were imaged at $0.5 \mu \mathrm{m} z$-plane intervals over $15-25$ $z$ sections. G4 foci counts for samples were analysed using the open-source image processing package $\mathrm{FIJI}^{29}$. The $Z$-stack images were converted to a single-plane maximum intensity projection (MIP) to accurately count the G4 foci. Thresholding was applied using appropriate levels to the MIP of the red channel (with G4 foci) to further reduce noise before doing an automated particle count using FIJI. G4 foci were counted for each individual nucleus in the images. A range of 10-178 nuclei were counted for each condition, and overlapping nuclei were excluded from analysis. The counts were analysed with one-way ANOVA with Tukey's test using GraphPad Prism statistical analysis software (GraphPad Software).

\section{Assessment for G4-forming potential in quantitative real-time PCR} candidate genes. Candidate genes ( $M Y C, B C L 2, H N R P A B)$ were screened with the QuadBase2 database ${ }^{30}$ using the following parameters: PG4 motif parameters $>$ Medium stringency $\left(\mathrm{G}_{3} \mathrm{~L}_{1-7}\right)$, Algorithm $>$ 'Greedy', Strands to search $>$ ' + ' and ' - ' strand, bulge size $>0$. Gene features queried were 'Gene body' and 'Around transcription start sites $(+/-500 \mathrm{bp})$ '.

Quantitative real-time PCR. HEK-293T and MCF-7 cells were seeded at 1.5 and $2.5 \times 10^{5}$ cells per well, respectively, in six-well plates overnight, before incubation with GNRs suspensions ( 0 or $100 \mu \mathrm{g} \mathrm{ml}^{-1}$ final concentration of nanorods) made up in fresh cell media. GQC-05 was also added to selected wells at the cell lines' corresponding $\mathrm{IC}_{50}$ doses. Cells were incubated for $48 \mathrm{~h}$ before being washed and collected for RNA isolation (RNeasy Mini kit; Qiagen) followed by reverse transcription to cDNA (Quantitect Reverse Transcription kit; Qiagen). Subsequently, quantitative real-time (qRT) PCR detection of MYC (NM_002467, Quantitect Primer Assay; Qiagen), BCL2 (NM_000633, Quantitect Primer Assay; Qiagen), HNRPAB (NM_004499, NM_031266, Quantitect Primer Assay; Qiagen), GAPDH (NM_001256799, NM_002046, NM_001289745, NM_001289746, Quantitect Primer Assay; Qiagen) and ACTB (NM_001101, XM_006715764, Quantitect Primer Assay; Qiagen) was performed on a LightCycler 480 (Roche). Changes in expression of $M Y C, B C L 2$ and $H N R P A B$ were normalized to both $G A P D H$ and $A C T B$ and calculated using the $\triangle \triangle C$ t method. Data were analysed with one-way ANOVA using the GraphPad Prism statistical analysis software (GraphPad Software)

CD spectroscopy. CD spectra were obtained using a Jasco J-810 spectropolarimeter with samples placed in quartz cuvette with $1 \mathrm{~mm}$ path length. Spectra were recorded in triplicate at room temperature at a scan rate of $100 \mathrm{~nm} \mathrm{~min}^{-1}$, with a response time of $1 \mathrm{~s}$, over a wavelength range of $230-330 \mathrm{~nm}$. Compounds ( $\mathrm{MTAB}, \mathrm{PPh}_{3}, \mathrm{PPh}_{3} \cdot \mathrm{AuCl}$ ) were dissolved in ultrapure water to a final concentration of the molar equivalent to the oligos tested. MTAB-GNRs was suspended to $1 \mu \mathrm{g} \mathrm{ml}^{-1}$ final concentration in ultrapure water. The control groups were suspended in only ultrapure water. All G4 oligonucleotide stocks were diluted to a final concentration of $5 \mu \mathrm{M}$ in $50 \mathrm{mM}$ Tris- $\mathrm{HCl}, \mathrm{pH} 7.4$, containing $50 \mathrm{mM} \mathrm{K}^{+}(\mathrm{KCl})$. The oligos were subsequently heated at $95^{\circ} \mathrm{C}$ for $10 \mathrm{~min}$ and slowly cooled to room temperature to induce $\mathrm{G} 4$ formation. This was followed by a 30 min incubation with compounds and MTAB-GNRs, respectively, to monitor interactions.

The maximum molar ellipticity for the $\mathrm{G} 4(262 \mathrm{~nm})$ was recorded for melting curves as the sample was heated from 20 to $95^{\circ} \mathrm{C}$ at a rate of $1{ }^{\circ} \mathrm{C} \mathrm{min}{ }^{-1}$. Melting temperature values were determined with GraphPad Prism statistical analysis software (GraphPad Software) within $\pm 1^{\circ} \mathrm{C}$. The oligo sequences used for CD were as follows:

MYC: 5' -TGGGGAGGGTGGGGAGGGTGGGGAAGG-3'

BCL2: 5' -AGGGGCGGGCGCGGGAGGAAGGGGGCGGGAGCGGGGC -3'

TEM preparation and imaging. HEK-293T and MCF-7 cells treated with $100 \mu \mathrm{g} \mathrm{ml}^{-1}$ of GNPs/GNRs and incubated at $37^{\circ} \mathrm{C}, 5 \% \mathrm{CO}_{2}$ for $48 \mathrm{~h}$ were trypsinized, fixed in $2 \%$ paraformaldehyde and stained with $1 \%$ osmium (ProSciTech) post-fixing for TEM analysis. This was followed by dehydration of the cells through an ethanol series to propylene oxide. The dehydrated samples were then embedded in a Procure-Araldite epoxy resin (ProSciTech) and cured for $24 \mathrm{~h}$. The resin was subsequently sectioned at $150 \mathrm{~nm}$ thickness and mounted onto carbon-coated copper TEM grids for imaging using JEOL 2100 at an accelerating voltage of $120 \mathrm{kV}$.

X-ray photoelectron spectroscopy. HEK-293T and MCF-7 cells were cultured and treated with GNPs or GNRs at $100 \mu \mathrm{g} \mathrm{ml}^{-1}$ as described earlier. After incubation for $48 \mathrm{~h}$, culture medium from each sample was collected and kept aside before the cells were washed with PBS and trypsinized. The trypsinized cells were collected and centrifuged at $200 \mathrm{~g}$ for $5 \mathrm{~min}$, followed by a final wash with PBS. The cells and their corresponding culture medium were flash frozen in liquid $\mathrm{N}_{2}$ and lyophilized for XPS measurements.

For gold leaching XPS experiments, MCF-7 cells were seeded overnight at a density of $2.5 \times 10^{5}$ cells per well in six-well plates, and treated with GNRs with either MTAB or CTAB surface coating $\left(100 \mu \mathrm{g} \mathrm{ml} \mathrm{m}^{-1}\right.$ made up in fresh cell medium) for a further $48 \mathrm{~h}$. At the designated time point, cells were washed thoroughly with PBS, trypsinized and counted. Cells were centrifuged ( $200 g$ for $5 \mathrm{~min}$ ) and the pellet washed with PBS before being centrifuged again and the supernatant carefully removed. Cells were resuspended in MilliQ $(500 \mu \mathrm{l})$ and sonicated until samples were homogenized $(4 \mathrm{~W}, 10 \mathrm{~s})$. Samples were centrifuged $(16,000 \mathrm{~g}$, $10 \mathrm{~min})$ to pellet GNR and cell debris before the supernatant $(450 \mu \mathrm{l})$ was carefully transferred to an Eppendorf tube, and the sample was concentrated via lyophilization on a centrifuge. Conditions were tested in triplicate, with untreated cells $(n=3)$. Samples were dissolved in and transferred to XPS sample grids with isopropanol.

XPS measurements were performed on a Kratos Axis Ultra DLD spectrometer using a monochromatic $\mathrm{AlK} \alpha(1,486.6 \mathrm{eV})$ irradiation source operated at $225 \mathrm{~W}$, a charge neutralizer and a hybrid lens system with magnetic lens providing an analysis area constrained to a $110-\mu \mathrm{m}$-diameter spot. All freeze-dried samples were pressed onto double-sided adhesive tape before introducing them into the ultrahigh vacuum of the analysis chamber. An argon gas cluster ion source operating at $20 \mathrm{keV}$ in $\mathrm{Ar}_{500}$ cluster mode was used to remove a thin overlayer of serum that initially obscured the Au $4 f$ signal. The vacuum pressure of the analysis chamber of the spectrometer was maintained at $5 \times 10^{-9}$ torr or lower throughout the duration of the analyses. The electron binding energy scale was referenced to the $\mathrm{C} 1 \mathrm{~s}$ line of aliphatic carbon, set at $285 \mathrm{eV}$. Differential surface charging of the samples was ruled out by checking the reproducibility of XPS spectra in repeated scans under varied X-ray exposure. XPS spectra were collected with a pass energy of $160 \mathrm{eV}$ for the survey spectra and $20 \mathrm{eV}$ for the high-resolution spectra. Data files were processed using CasaXPS software and interpreted using relative sensitivity factors provided by the instrument manufacturer (Kratos) as a guide. The relative atomic concentrations of MCF-7 cells (non-treated control and MTAB-NRS treated) were calculated from the survey spectra using the areas of the background-subtracted $\mathrm{Au} 4 f, \mathrm{Na} 1 s, \mathrm{~K} 2 p, \mathrm{C} 1 s, \mathrm{~N} 1 s$ and $\mathrm{O} 1 s$ photoemission signals. Background subtractions using a Shirley background were applied to all high-resolution spectra. Au $4 f$ spectra were fitted using a hybrid Doniach-Sunjic line shape convoluted with a Gaussian/Lorentzian sum for metallic Au, while Gaussian-Lorentzian line shapes were used to fit higher-oxidation-state Au species. The area ratios for the Au 4f $f_{5 / 2}$ : Au 4f $f_{7 / 2}$ doublets were set to 3:4, and the FWHM values were constrained to values considered reasonable for each chemical state.

NanoSIMS ion map acquisition. HEK-293T and MCF-7 cells were fixed and embedded in resin according to the protocol for TEM preparation. Subsequently 1 - $\mu$ m-thick sections were placed onto clean silicon wafers and coated with $\sim 5 \mathrm{~nm}$ of carbon for imaging using the CAMECA NanoSIMS 50 under a $\mathrm{Cs}^{+}$primary ion beam with a total impact energy of $16 \mathrm{keV}$ as described in the previous report ${ }^{31}$. The mass spectrometer was tuned to detect ${ }^{12} \mathrm{C}^{14} \mathrm{~N}^{-},{ }^{31} \mathrm{P}^{-}$and ${ }^{197} \mathrm{Au}$ ions simultaneously. The position of the ${ }^{197} \mathrm{Au}$ mass peak was calibrated using gold foil in the absence of cells, and the mass spectrometer tuned to high mass resolution to avoid isobaric interferences on mass 197 (for example, ${ }^{133} \mathrm{Cs}^{32} \mathrm{~S}_{2}$ ). Survey images were initially acquired at low resolution over a $60 \times 60 \mu \mathrm{m}^{2}$ area with a corresponding pixel resolution of $512 \times 512$ pixels using a primary beam diameter of $\sim 110 \mathrm{~nm}$ (low-resolution images) at a beam current of $1-2 \mathrm{pA}$. For high-resolution images, individual cells were scanned over a $18 \times 18 \mu \mathrm{m}^{2}$ area, at a resolution of $256 \times 256$ pixels, with a dwell time of $50 \mathrm{~ms}$ per pixel using a beam diameter of $\sim 50 \mathrm{~nm}$ (high-resolution images) at $0.1-0.2 \mathrm{pA}$. Dwell times were kept constant across samples imaged at the same resolution. The ion signal was detected and counted by an electron multiplier at each pixel position in the image. Images were corrected for a detector deadtime of $44 \mathrm{~ns}$. Image processing was carried out using FIJI (ImageJ) ${ }^{29}$ and the OpenMIMS ImageJ plugin (http://nano.bwh.harvard. edu/openmims)

The NanoSIMS generates 32-bit images consisting of the number of secondary ion counts recorded for each pixel coordinate. Pixels in the areas corresponding to the GNRs typically record ion counts in the thousands. Analysis of control samples containing no GNR determined that a diffuse, low-level background signal of 1-2 counts per pixel was always present. For illustration purposes (as shown in Supplementary Fig. 5), the contrast and brightness of ${ }^{197} \mathrm{Au}^{-}$images were thresholded to highlight the low intensity signal corresponding to the diffusion of $\mathrm{Au}$ ions from the GNR. This was achieved by setting the maximum pixel 
count to 4 , and the minimum pixel count to 2, which enhances the Au diffusion signal while suppressing the low-level background signal. ${ }^{31} \mathrm{P}^{-}$images clearly show the nuclear envelopes and were used as guides to demarcate the nuclear region in the ${ }^{12} \mathrm{C}^{14} \mathrm{~N}^{-}$images. Un-thresholded ${ }^{197} \mathrm{Au}^{-}$images were overlaid on the thresholded ${ }^{197} \mathrm{Au}^{-}$images to show regions of GNRs and Au ions diffused out from the GNRs, respectively. These images were subsequently overlaid on top of their corresponding ${ }^{12} \mathrm{C}^{14} \mathrm{~N}^{-}$images. The same method of processing was applied to all samples. This way of illustrating Au diffusion does not modify the number of counts recorded per pixel in any way and is only an aid to visualization.

\section{Data availability}

The data that support the plots within this paper and other findings of this study are available from the corresponding authors upon reasonable request.

\section{References}

27. Jana, N. R., Gearheart, L. \& Murphy, C. J. Seeding growth for size control of 5-40 nm diameter gold nanoparticles. Langmuir 17, 6782-6786 (2001).

28. Ye, X., Zheng, C., Chen, J., Gao, Y. \& Murray, C. B. Using binary surfactant mixtures to simultaneously improve the dimensional tunability and monodispersity in the seeded growth of gold nanorods. Nano Lett. 13, 765-771 (2013).

29. Schindelin, J. et al. Fiji: an open-source platform for biological-image analysis. Nat. Methods 9, 676-682 (2012).

30. Dhapola, P. \& Chowdhury, S. QuadBase2: web server for multiplexed guanine quadruplex mining and visualization. Nucleic Acids Res. 44, W277-W283 (2016).

31. Wedlock, L. E. et al. NanoSIMS multi-element imaging reveals internalisation and nucleolar targeting for a highly-charged polynuclear platinum compound. Chem. Commun. 49, 6944-6946 (2013). 


\section{Reporting Summary}

Nature Research wishes to improve the reproducibility of the work that we publish. This form provides structure for consistency and transparency in reporting. For further information on Nature Research policies, see Authors \& Referees and the Editorial Policy Checklist.

\section{Statistical parameters}

When statistical analyses are reported, confirm that the following items are present in the relevant location (e.g. figure legend, table legend, main text, or Methods section).

$\mathrm{n} / \mathrm{a} \mid$ Confirmed

$\bigotimes$ The exact sample size $(n)$ for each experimental group/condition, given as a discrete number and unit of measurement

$\square$ An indication of whether measurements were taken from distinct samples or whether the same sample was measured repeatedly

The statistical test(s) used AND whether they are one- or two-sided

Only common tests should be described solely by name; describe more complex techniques in the Methods section.

$\searrow$ A description of all covariates tested

$\square$ A description of any assumptions or corrections, such as tests of normality and adjustment for multiple comparisons

$\square$ A full description of the statistics including central tendency (e.g. means) or other basic estimates (e.g. regression coefficient) AND

variation (e.g. standard deviation) or associated estimates of uncertainty (e.g. confidence intervals)

For null hypothesis testing, the test statistic (e.g. $F, t, r$ ) with confidence intervals, effect sizes, degrees of freedom and $P$ value noted

Give $P$ values as exact values whenever suitable.

Х $\square$ For Bayesian analysis, information on the choice of priors and Markov chain Monte Carlo settings

Х $\square$ For hierarchical and complex designs, identification of the appropriate level for tests and full reporting of outcomes

Х $\square$ Estimates of effect sizes (e.g. Cohen's $d$, Pearson's $r$ ), indicating how they were calculated

$\varnothing$ Clearly defined error bars

State explicitly what error bars represent (e.g. SD, SE, Cl)

Our web collection on statistics for biologists may be useful.

\section{Software and code}

Policy information about availability of computer code
Data collection
No software was used.

Data analysis

Graphpad Prism v6.01, FIJI (ImageJ v1.51e)

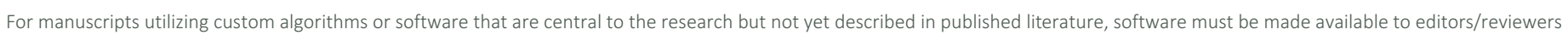

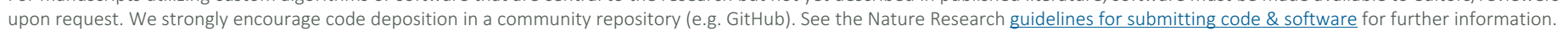

\section{Data}

Policy information about availability of data

All manuscripts must include a data availability statement. This statement should provide the following information, where applicable:

- Accession codes, unique identifiers, or web links for publicly available datasets

- A list of figures that have associated raw data

- A description of any restrictions on data availability

The data that support the plots within this paper and other findings of this study are available from the corresponding authors upon reasonable request. 
Please select the best fit for your research. If you are not sure, read the appropriate sections before making your selection.

$\bigotimes$ Life sciences $\quad \square$ Behavioural \& social sciences

For a reference copy of the document with all sections, see nature.com/authors/policies/Reportingsummary-flat.pdf

\section{Life sciences}

\section{Study design}

All studies must disclose on these points even when the disclosure is negative.

Sample size No sample-size calculation was performed. For each experiment at least 3 technical replicates for each condition was performed to test the variability in the experimental protocol and 2 biological replicates to control for biological variations (Biological replicates were not performed for CD spectroscopy and ICP analyses). 2 biological replicates were chosen and were deemed sufficient as stated in the experimental guidelines recommended by the ENCODE Consortium.

Data exclusions No data was excluded.

Replication 2 biological replicates (all experiments except CD spectroscopy and ICP analyses) were performed in total by different experimenters, with each biological replicate having at least 3 technical replicates. Results from both biological replicates were included in the manuscript. All replications were successful.

Randomization No randomization was performed with the samples. The cells in each well/plate were equally viable and indistinguishable from each other. Additionally, all cell-based experiments were replicated with 2 different experimenters.

Blinding A single-blind study was performed for the collection and analyses of G4 foci counts. The other experiments were not performed in blind as they were mainly conducted by a single experimenter per replication.

\section{Materials \& experimental systems}

Policy information about availability of materials

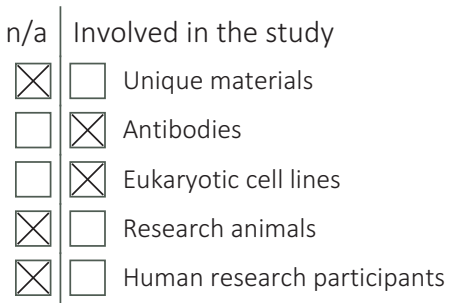

\section{Antibodies}

Antibodies used

FLAG-tagged BG4 antibody expressed from pSANG10-3F-BG4 (1uM final concentration of antibody, Addgene plasmid \#55756, low copy), anti-FLAG secondary antibody (1/1000 dilution, catalog \#2368, Lot \#12, Cell Signaling Technology), anti-rabbit Alexa Fluor ${ }^{\circledast 5} 59$ tertiary antibody (1/2000 dilution, catalog A-11037, Lot 1915874, ThermoFisher Scientificl.

Validation

Expressed BG4 was compared and validated against the commercially-available BG4 (\#MABE917, Merck) using SDS-PAGE. Control experiments with secondary and tertiary antibodies in the absence of primary antibody were performed to confirm specificity of secondary antibody to BG4. Anti-FLAG and Alexa Fluor 594 antibodies were not modified in any way and used as received from the manufacturers.

Eukaryotic cell lines

Policy information about cell lines

Cell line source(s)

Authentication

Mycoplasma contamination

Commonly misidentified lines (See ICLAC register)
MCF-7 and HEK-293T were sourced from ATCC.

None of the cell lines were authenticated as they were sourced from ATCC.

All cell lines were tested negative for mycoplasma contamination.

MCF-7, HEK-293T. MCF-7 was selected for use as it has been previously utilised in the experiments involving G4 and GQC-05. 
Method-specific reporting

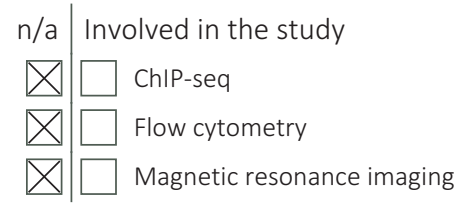

3
$\vdots$
$\vdots$
5
$\tilde{0}$
0

3 
In the format provided by the authors and unedited.

\title{
Intracellular speciation of gold nanorods alters the conformational dynamics of genomic DNA
}

\author{
Diwei Ho ${ }^{1,12}$, Jessica A. Kretzmann ${ }^{1,12}$, Marck Norret', Priyanka Toshniwal', Jean-Pierre Veder' \\ Haibo Jiang ${ }^{1,3}$, Paul Guagliardo ${ }^{3}$, Alaa M. Munshi ${ }^{111}$, Reena Chawla ${ }^{4,5,6}$, Cameron W. Evans', \\ Tristan D. Clemons', Michelle Nguyen', Amy L. Kretzmann', Amanda J. Blythe', Martin Saunders ${ }^{1}{ }^{13}$, \\ Michael Archer ${ }^{7}$, Melinda Fitzgerald ${ }^{7,8,9}$, Jeffrey A. Keelan ${ }^{10}{ }^{10}$, Charles S. Bond ${ }^{10}{ }^{1}$, Matt R. Kilburn ${ }^{(3}{ }^{3}$, \\ Laurence H. Hurley $4,5,6$, Nicole M. Smith ${ }^{1 \star}$ and K. Swaminathan lyer ${ }^{1 \star}$
}

\footnotetext{
'School of Molecular Sciences, The University of Western Australia, Perth, Western Australia, Australia. ${ }^{2}$ John de Laeter Centre, Curtin University, Perth, Western Australia, Australia. ${ }^{3}$ Centre for Microscopy, Characterisation and Analysis, The University of Western Australia, Perth, Western Australia, Australia. ${ }^{4}$ College of Pharmacy, University of Arizona, Tucson, AZ, USA. ${ }^{5}$ BIO5 Institute, University of Arizona, Tucson, AZ, USA. ${ }^{6}$ Arizona Cancer Center, University of Arizona, Tucson, AZ, USA. ${ }^{7}$ School of Biological Sciences, The University of Western Australia, Perth, Western Australia, Australia. ${ }^{8} \mathrm{Curtin}$ Health Innovation Research Institute, Curtin University, Perth, Western Australia, Australia. ${ }^{9}$ Perron Institute for Neurological and Translational Science, Sarich Neuroscience Research Institute, Perth, Western Australia, Australia. ${ }^{10}$ Schools of Obstetrics \& Gynaecology and Biomedical Sciences, The University of Western Australia, Perth, Western Australia, Australia. "Present address: Department of Chemistry, Faculty of Applied Science, Umm Al-Qura University, Makkah, Saudi Arabia. ${ }^{12}$ These authors contributed equally: Diwei Ho, Jessica A. Kretzmann. *e-mail: nicole.smith@uwa.edu.au; swaminatha.iyer@uwa.edu.au
} 


\section{Supplementary results}

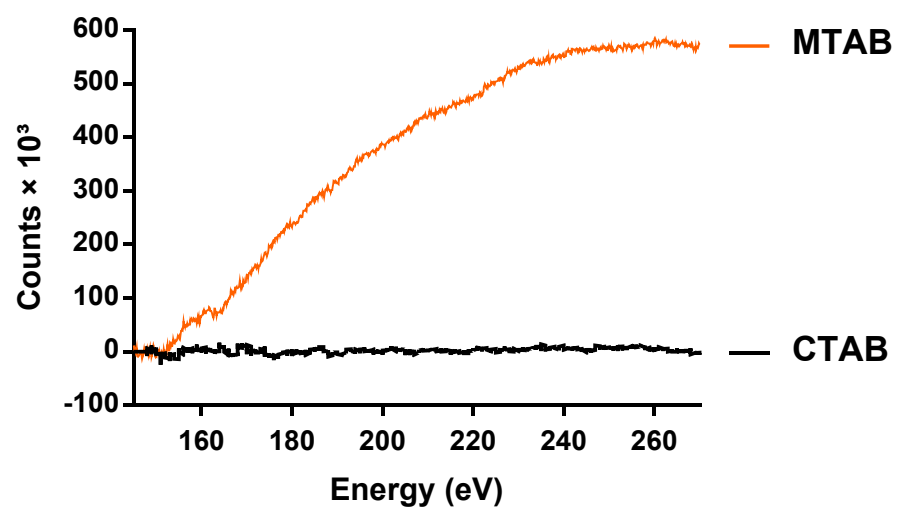

Supplementary Figure S1. The Electron Energy Loss Spectra (EELS) of the S L2,3-edge after background subtraction. No signal above the background for $\mathrm{S}$ was observed for CTABGNRs, whereas for MTAB-GNRs a clear $\mathrm{S} \mathrm{L}_{2,3}$ peak was revealed consistent in both energy and structure with that reported in the Gatan EELS Atlas, after 6 washes. ${ }^{8}$

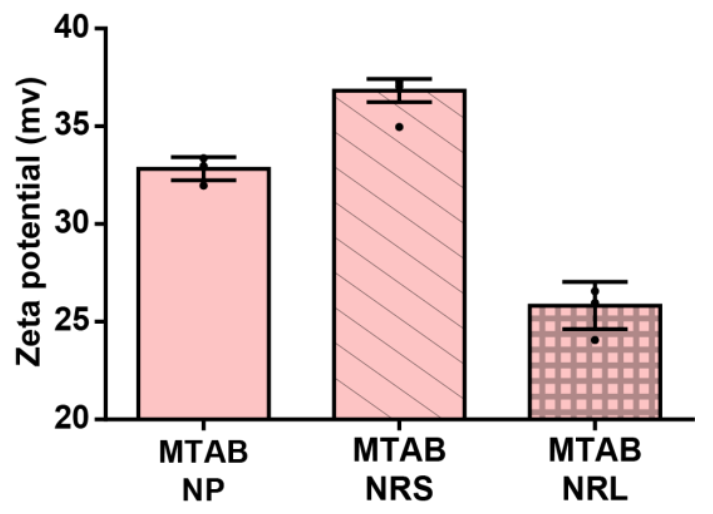

Supplementary Figure S2. Zeta potentials of MTAB NP, NRS and NRL. All data $(\mathrm{n}=3$ per condition) in graphs are represented as mean \pm SD and individual data points shown as dot plots. 
a

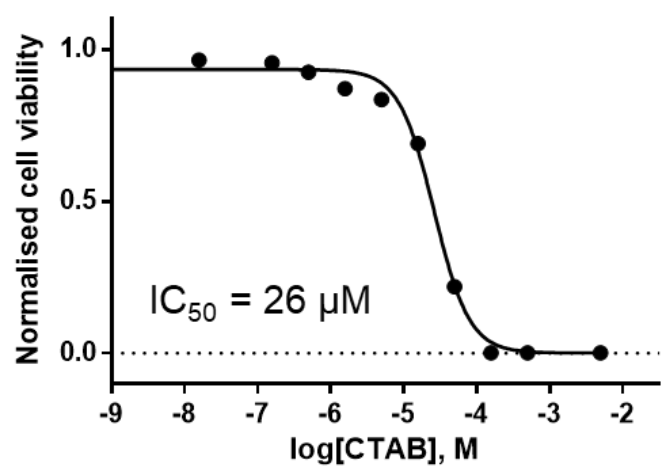

b

MCF-7 (MTAB)

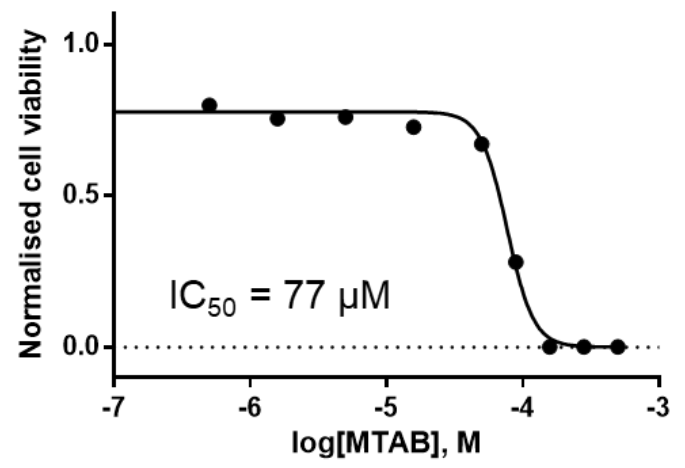

Supplementary Figure S3. IC $_{50}$ curves of free (a) CTAB and (b) MTAB in MCF-7. Cell viability was assessed over $48 \mathrm{~h}$ for all samples.

a

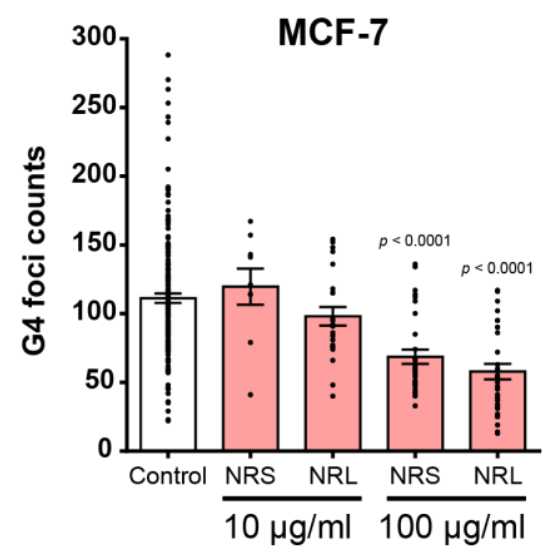

b

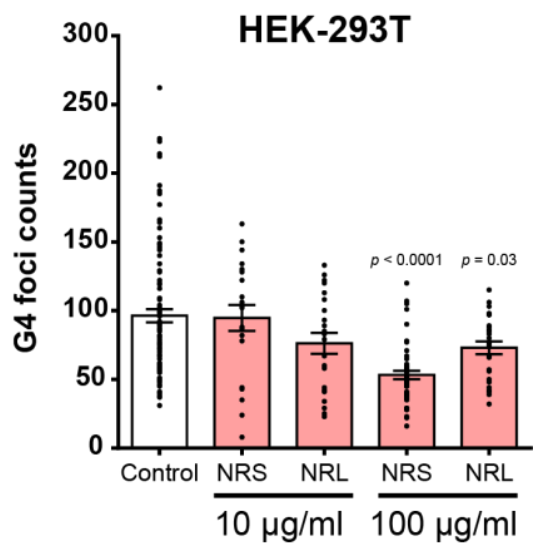

Supplementary Figure S4. G4 foci counts in (a) MCF-7 and (b) HEK-293T after exposure to NRS/NRL at $10 \mu \mathrm{g} / \mathrm{ml}$ and $100 \mu \mathrm{g} / \mathrm{ml}$ after $48 \mathrm{~h}$. 1-way ANOVA statistical test was performed against control for (a \& b). All data ( $\mathrm{n}=10$ to 178 nuclei per condition from 3 biological replicates) in graphs are represented as mean \pm SEM and individual data points shown as dot plots 

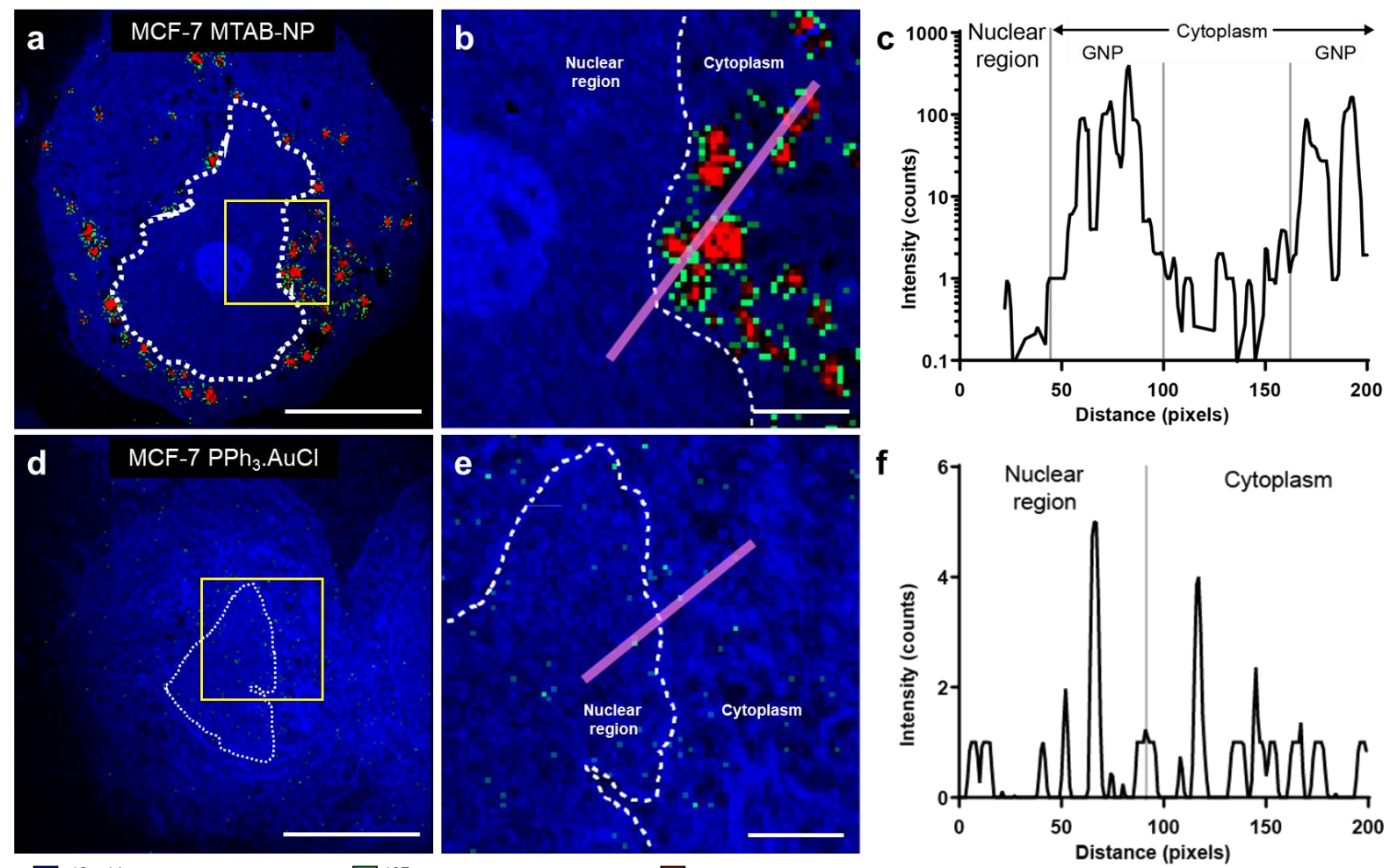

${ }^{12} \mathrm{C}^{14} \mathrm{~N} \cdot$ elemental map

${ }^{197} \mathrm{Au}^{-}$elemental map

GNR clusters

Supplementary Figure S5. Merged nanoscale secondary ion mass spectrometry elemental maps of representative MTAB-NP (a \& b) and $\mathrm{PPh}_{3}$.AuCl-treated (d \& e) MCF-7 samples. Nuclear region is highlighted with white dotted line, magnified regions of interest in (a) and (d) are indicated by the yellow boxes and are shown in (b) and (e) respectively. No Au signals (green) were observed within the nuclei of MTAB-NP treated MCF-7 cells. Au signals were sparsely dispersed within the nuclei and cytoplasm of $\mathrm{PPh}_{3}$. AuCl treated MCF-7 cells. Representative intensity profiles (c \& f) of the diffused Au signals within the nuclei obtained from regions of interest represented by the pink line in (b) and (e). Intensity counts below 2 was considered as background noise. Experiments were repeated twice independently, with similar results. Scale bars: (a \& d) $5 \mu \mathrm{m}$; (b \& e) $1 \mu \mathrm{m}$. 

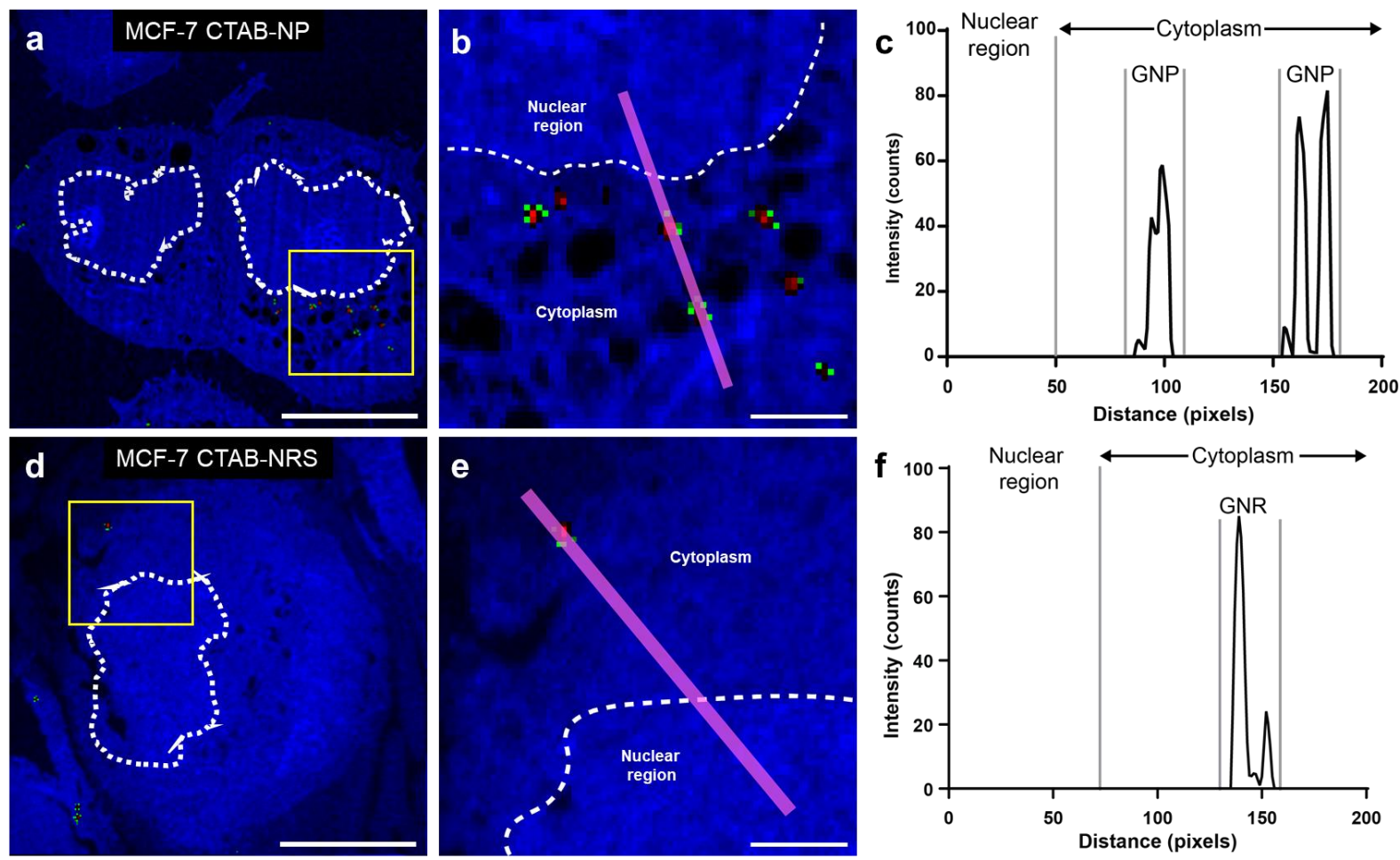

${ }^{12} \mathrm{C}^{14} \mathrm{~N}$ - elemental map

${ }^{197} \mathrm{Au}-$ elemental map

GNR clusters

Supplementary Figure S6. Merged nanoscale secondary ion mass spectrometry elemental maps of representative CTAB-NP (a) and CTAB-NRS treated (b) MCF-7 samples. Nuclear region is highlighted with white dotted line, magnified regions of interest in (a) and (d) are indicated by the yellow boxes and are shown in (b) and (e) respectively. No Au signals (green) were observed within the nuclei of CTAB-NP/NRS treated MCF-7 cells. Representative intensity profiles (c \& f) of the diffused Au signals within the nuclei obtained from regions of interest represented by the pink line in (b) and (e). Intensity counts below 2 was considered as background noise. Experiments were repeated twice independently, with similar results. Scale bars: (a \& d) $5 \mu \mathrm{m}$; (b \& e) $1 \mu \mathrm{m}$. 


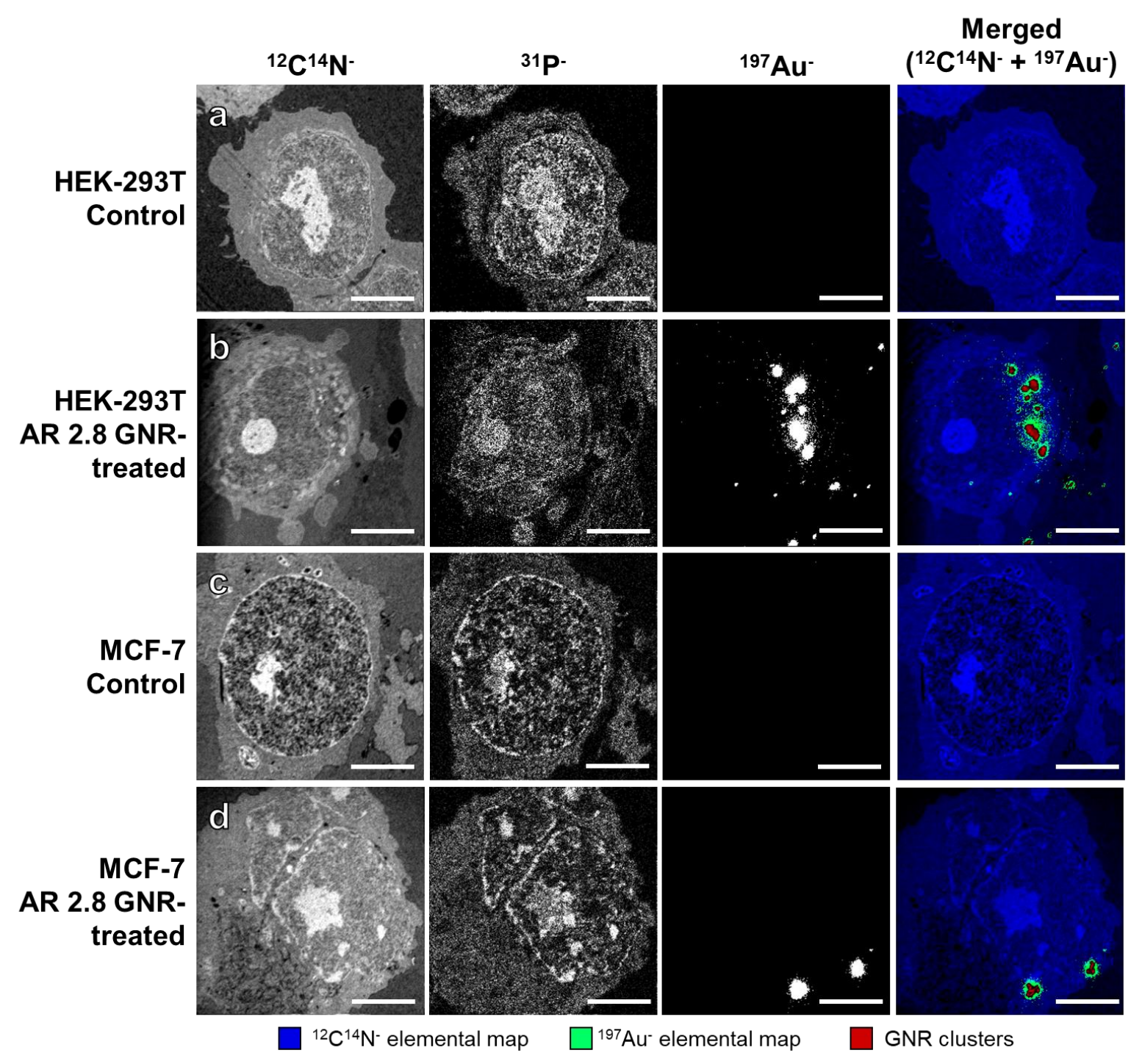

Supplementary Figure S7. Elemental maps of HEK-293T and MCF-7 control and treated samples visualised by nanoscale secondary ion mass spectrometry. Contrast and brightness on ${ }^{197} \mathrm{Au}^{-}$images have been adjusted to show low concentrations of $\mathrm{Au}$ signals but not background signal. The location of GNRS are shown in red. These adjustments were performed for all NanoSIMS images. Scale bar: $5 \mu \mathrm{m}$. 

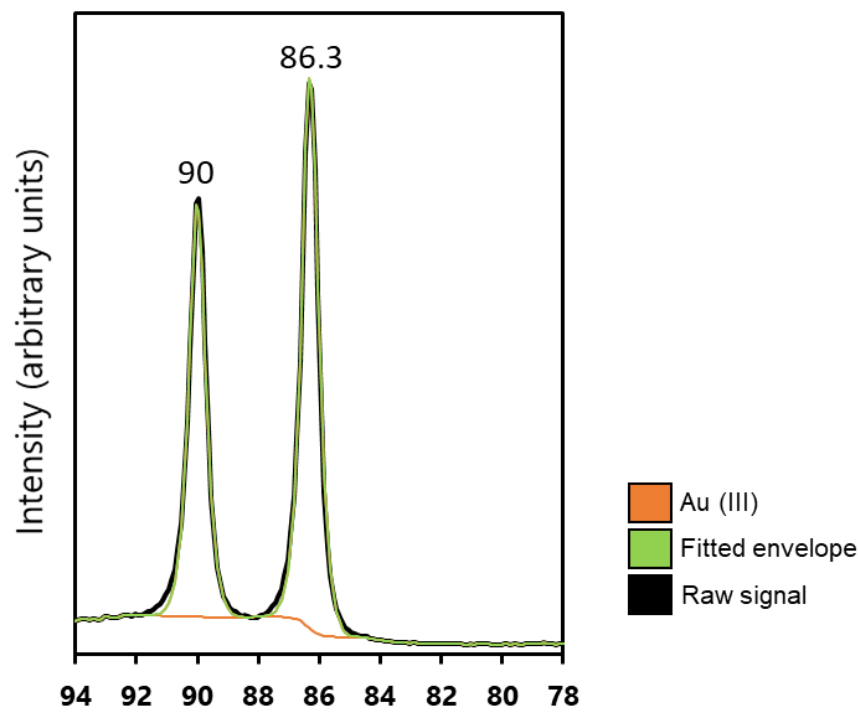

Supplementary Figure S8. XPS spectra of the supernatant from samples with MTAB-NRS exposed to MCF-7.

a

HEK-293T (GQC-05)

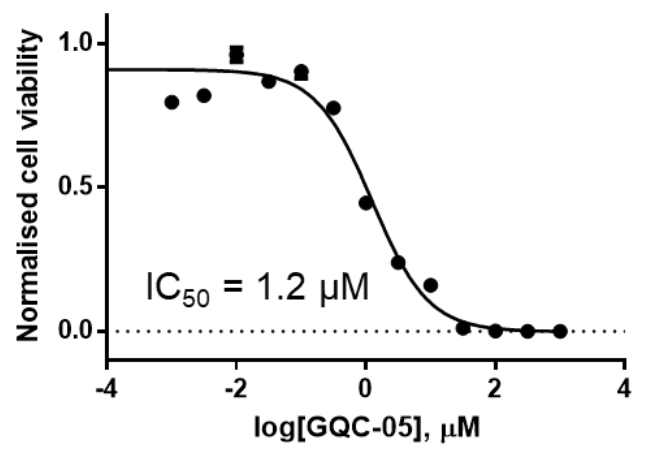

b

MCF-7 (GQC-05)

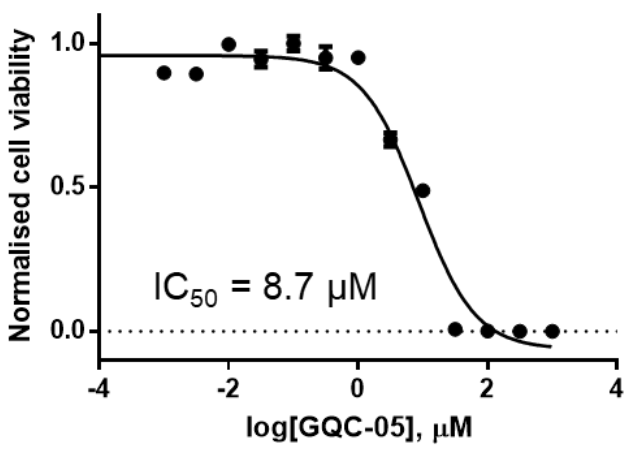

Supplementary Figure S9. IC 50 curves of GQC-05 in (a) HEK-293T and (b) MCF-7. Cell viability was assessed over $48 \mathrm{~h}$ for all samples. 